Supporting information

\title{
Salt formation: route to improve energetic performance and molecular stability simultaneously
}

Qian Wang, ${ }^{\dagger}$ Yanli Shao, ${ }^{\dagger}$ Ming Lu ${ }^{*, \dagger}$

${ }^{\dagger}$ School of Chemical Engineering, Nanjing University of Science and Technology, Nanjing 210094, China.

${ }^{\ddagger}$ Key Laboratory of Healthy \& Intelligent Kitchen System Integration of Zhejiang Province, No.218 Binhai 2 Road, Hangzhou Bay New District, Cixi, Ningbo, China.

Table of Contents

1. Experimental section

2. Computational Details

3. Crystallographic data for compounds $2-8$

4. IR and NMR spectra

5. DSC curves

6. References 


\section{Experimental section}

\section{General Methods}

All reagents were of analytical grade and were used without further purification. Elemental analyses were performed on a vario EL III CHNOS elemental analyzer. ${ }^{1} \mathrm{H},{ }^{13} \mathrm{C}$ NMR spectra were recorded on nuclear magnetic resonance spectrometer operating at 500 and $50.69 \mathrm{MHz}$, respectively. Chemical shifts in the ${ }^{1} \mathrm{H}$ and ${ }^{13} \mathrm{C}$ spectra were reported in ppm relative to TMS. The DSC spectra were obtained on a differential scanning calorimeter (Mettler Toledo DSC823e) at a heating rate of $5{ }^{\circ} \mathrm{C} \mathrm{min}^{-1}$. TGA were also performed at a heating rate of $5{ }^{\circ} \mathrm{C} \mathrm{min}^{-1}$ in flowing high-purity nitrogen on a Mettler Toledo TGA/SDTA851 $1^{\mathrm{e}}$ instrument. FT-IR spectra were obtained on a Thermo Nicolet iS 10 spectrometer. Powder X-ray diffraction (PXRD) measurements were performed on a Bruker D8 Advance X-ray diffractometer using $\mathrm{Cu} K \alpha(\lambda=1.5406 \AA)$ radiation.

\section{X-ray crystallography}

The data for 2-8 was collected with a Bruker SMART APEX II CCD diffractometer with graphite-monochromated Mo-Ka radiation ( $\lambda=0.071073$ $\mathrm{nm}$ ) at $172-173 \mathrm{~K}$. The data collection and the initial unit cell refinement were performed by using APEX2 (v2010.3-0). Data Reduction was performed by using SAINT (v7.68A) and XPREP (v2008/2). Empirical absorption corrections were applied by using the SADABS (v2008/1) program. The structures were solved by direct methods and refined by the full matrix least-squares based on F2 using SHELXTL--2014/7 (Sheldrick, 2014) programme package. All non-hydrogen atoms were refined anisotropically. The hydrogen atoms attached to ligands were generated geometrically and refined using a riding model.

\section{Syntheses}

Caution! Although all the fused compounds obtained here have a good sensitivity, they should be handled carefully. Some safety practic es must be provided, such as face shield, leather gloves.

3-(4-amino-1,2,5-oxadiazol-3-yl)-1,2,4-oxadiazol-5-amine (1) ${ }^{1}$ was synthesized based on the reported method.

N-(4-(5-amino-1,2,4-oxadiazol-3-yl)-1,2,5-oxadiazol-3-yl)nitramide (2): Compound $\mathbf{1}$ (0.500 g, $2.97 \mathrm{mmol})$ was slowly added to $1 \mathrm{~mL}$ fuming nitric acid at $0{ }^{\circ} \mathrm{C}$. The reaction mixture was kept for $3 \mathrm{~h}$ at this temperature. Then the resulting solution was poured onto $50 \mathrm{~g}$ ice-water and a white solid was formed. The precipitate was filtered off, washed with water and dried in air afford compound $\mathbf{1}(0.443 \mathrm{~g}, 2.08 \mathrm{mmol})$ in a yield of $70 \%$. $T_{\mathrm{d}}: 111{ }^{\circ} \mathrm{C} .{ }^{1} \mathrm{H}$ NMR $\left(500 \mathrm{MHz}, \mathrm{DMSO}-d_{6}\right): \delta 8.30(\mathrm{~s}, 2 \mathrm{H}), 13.59(\mathrm{~s}, 1 \mathrm{H}) \mathrm{ppm} .{ }^{13} \mathrm{C}$ NMR $\left(500 \mathrm{MHz}, \mathrm{DMSO}-d_{6}\right): \delta 142.68,148.38,157.23,172.04$ ppm. IR (KBr): $\tilde{v} 3381,3257,3186,1690,1665,1588,1503,1306,1158,969,915,808,727 \mathrm{~cm}^{-1}$. Elemental analysis for $\mathrm{C}_{4} \mathrm{H}_{3} \mathrm{~N}_{7} \mathrm{O}_{4}(213.11)$ : calcd C 22.54, H 1.42, N 46.01\%. Found: C 22.53, H 1.41, N 46.03\%.

\section{General procedure for the preparation of salts 3 to 8}

A solution of $28 \%$ aqueous ammonia $(0.024 \mathrm{~g}, 1.37 \mathrm{mmol}), 98 \%$ hydrazine monohydride $(0.511 \mathrm{~g}, 1.00 \mathrm{mmol}), 50 \%$ aqueous hydroxylamine $(0.661$ $\mathrm{mg}, 1 \mathrm{mmol})$, guanidine carbonate $(0.121 \mathrm{~g}, 1.00 \mathrm{mmol})$, carbohydrazide $(0.090 \mathrm{~g}, 1.00 \mathrm{mmol}), 4-\mathrm{NH}_{2}-1,2,4$-triazole $(0.084 \mathrm{~g}, 1.37 \mathrm{mmol})$ in ethanol $(5 \mathrm{~mL})$ were slowly added under stirring to a solution of $2(0.249 \mathrm{~g}, 1.00 \mathrm{mmol})$ in acetonitrile $(10 \mathrm{~mL})$ at $25^{\circ} \mathrm{C}$. After stirring for $2 \mathrm{~h}$, the precipitate was collected, washed with acetonitrile, and dried in air.

Ammonium (4-(5-amino-1,2,4-oxadiazol-3-yl)-1,2,5-oxadiazol-3-yl)(nitro)amide (3): Yield: $0.209 \mathrm{~g}, 0.91 \mathrm{mmol}, 91 \%$. White solid. $T_{\mathrm{d}}: 206^{\circ} \mathrm{C}$. ${ }^{1} \mathrm{H}$ NMR $\left(500 \mathrm{MHz}\right.$, DMSO- $\left.d_{6}\right): \delta 7.15(\mathrm{~s}, 4 \mathrm{H}), 8.15$ (s, 2H) ppm. ${ }^{13} \mathrm{C}$ NMR (500 MHz, DMSO- $\left.d_{6}\right): \delta 143.21,158.18,159.69,172.48 \mathrm{ppm}$. IR (KBr): $\tilde{v} 3589,3135,1701,1634,1511,1432,1398,1187,1054,971,887,829,773 \mathrm{~cm}^{-1}$. Elemental analysis for $\mathrm{C}_{4} \mathrm{H}_{6} \mathrm{~N}_{8} \mathrm{O}_{4}(230.14)$ : calcd C 20.88, $\mathrm{H} 2.63$, N 48.69\%. Found: C 20.86, H 2.65, N 48.68\%.

Hydrazinium (4-(5-amino-1,2,4-oxadiazol-3-yl)-1,2,5-oxadiazol-3-yl)(nitro)amide (4): Yield: $0.226 \mathrm{~g}, 0.92 \mathrm{mmol}, 92 \%$. White solid. $T_{\mathrm{d}}: 192{ }^{\circ} \mathrm{C}$. ${ }^{1} \mathrm{H}$ NMR $\left(500 \mathrm{MHz}\right.$, DMSO- $\left.d_{6}\right): \delta 7.10(\mathrm{~s}, 5 \mathrm{H}), 8.15$ (s, 2H) ppm. ${ }^{13} \mathrm{C}$ NMR $\left(500 \mathrm{MHz}\right.$, DMSO- $\left.d_{6}\right): \delta 142.98,157.89,159.59,172.48 \mathrm{ppm}$. IR $(\mathrm{KBr}):$ $\tilde{v} 3343,3131,2997,1659,1621,1503,1429,1177,1113,1098,967,771 \mathrm{~cm}^{-1}$. Elemental analysis for $\mathrm{C}_{4} \mathrm{H}_{7} \mathrm{~N}_{9} \mathrm{O}_{4}(245.16)$ : calcd C 19.60, H 2.88, N $51.42 \%$. Found: C $19.61, \mathrm{H} 2.89$, N 51.44\%.

Hydroxylammonium (4-(5-amino-1,2,4-oxadiazol-3-yl)-1,2,5-oxadiazol-3-yl)(nitro)amide (5): Yield: 0.219 g, 0.89 mmol, $89 \%$. White solid. $T_{\mathrm{d}}$ $175{ }^{\circ} \mathrm{C} .{ }^{1} \mathrm{H}$ NMR $\left(500 \mathrm{MHz}, \mathrm{DMSO}-d_{6}\right): \delta 8.15(\mathrm{~s}, 2 \mathrm{H}), 10.14(\mathrm{~s}, 4 \mathrm{H}) \mathrm{ppm} .{ }^{13} \mathrm{C}$ NMR $\left(500 \mathrm{MHz}, \mathrm{DMSO}-d_{6}\right): \delta 143.19,158.08,159.65,172.48 \mathrm{ppm}$. IR (KBr): $\tilde{v} 3597,3331,3146,1682,1602,1387,1370,1190,1073,1012,975,934,773 \mathrm{~cm}^{-1}$. Elemental analysis for $\mathrm{C}_{4} \mathrm{H}_{6} \mathrm{~N}_{8} \mathrm{O}_{5}(246.14)$ : calcd $\mathrm{C}$ 19.52, H 2.46, N 45.52\%. Found: C19.51, H 2.45, N 45.51\%.

Guanidinium (4-(5-amino-1,2,4-oxadiazol-3-yl)-1,2,5-oxadiazol-3-yl)(nitro)amide (6): Yield: $0.287 \mathrm{~g}, 0.93 \mathrm{mmol}, 93 \%$. White solid. $T_{\mathrm{d}}: 235{ }^{\circ} \mathrm{C}$. ${ }^{1} \mathrm{H}$ NMR $\left(500 \mathrm{MHz}, \mathrm{DMSO}-d_{6}\right): \delta 6.97(\mathrm{~s}, 6 \mathrm{H}), 8.14(\mathrm{~s}, 6 \mathrm{H}) \mathrm{ppm} .{ }^{13} \mathrm{C}$ NMR $\left(500 \mathrm{MHz}, \mathrm{DMSO}-d_{6}\right): \delta 143.38,158.21,158.27,159.70,172.50 \mathrm{ppm}$. IR (KBr): $\tilde{v} 3559,3451,3339,3141,1697,1676,1659,1500,1396,1182,1080,824,772, \mathrm{~cm}^{-1}$. Elemental analysis for $\mathrm{C}_{5} \mathrm{H}_{8} \mathrm{~N}_{10} \mathrm{O}_{4}(272.19)$ : calcd C 22.06, H 2.96, N 51.46\%. Found: C 22.07, H 2.97, N 51.47\%.

Diaminouronium (4-(5-amino-1,2,4-oxadiazol-3-yl)-1,2,5-oxadiazol-3-yl)(nitro)amide (7): Yield: $0.285 \mathrm{~g}, 0.94 \mathrm{mmol}, 94 \%$. White solid. $T_{\mathrm{d}}$ $190{ }^{\circ} \mathrm{C} .{ }^{1} \mathrm{H}$ NMR $\left(500 \mathrm{MHz}\right.$, DMSO- $\left.d_{6}\right): \delta 8.15$ (br) ppm. ${ }^{13} \mathrm{C}$ NMR $\left(500 \mathrm{MHz}\right.$, DMSO- $\left.d_{6}\right): \delta 143.09,158.02,159.45,159.60,172.47 \mathrm{ppm}$. IR (KBr): $\tilde{v} 3363,3139,2987,2705,1665,1626,1505,1489,1316,1296,1180,1096,971,825,793,773 \mathrm{~cm}^{-1}$. Elemental analysis for $\mathrm{C}_{5} \mathrm{H}_{9} \mathrm{~N}_{11} \mathrm{O}_{5}(303.20)$ : calcd C 19.81, H 2.99, N 50.82\%. Found: C 19.82, H 2.97, N 50.84\%.

4-Amino-1,2,4-triazolium (4-(5-amino-1,2,4-oxadiazol-3-yl)-1,2,5-oxadiazol-3-yl)(nitro)amide (8): Yield: $0.270 \mathrm{~g}, 0.91 \mathrm{mmol}, 91 \%$. White solid. $T_{\mathrm{d}}: 191{ }^{\circ} \mathrm{C} .{ }^{1} \mathrm{H}$ NMR $\left(500 \mathrm{MHz}, \mathrm{DMSO}-d_{6}\right): \delta 8.22(\mathrm{~s}, 2 \mathrm{H}), 9.31(\mathrm{~s}, 2 \mathrm{H}) \mathrm{ppm} .{ }^{13} \mathrm{C}$ NMR $\left(500 \mathrm{MHz}, \mathrm{DMSO}-d_{6}\right): \delta 143.21,144.36,156.58,159.36$, 172.52 ppm. IR (KBr): $\tilde{v} 3282,3129,1917,1666,1500,1398,1175,1047,969,886,814,771,617 \mathrm{~cm}^{-1}$. Elemental analysis for $\mathrm{C}_{6} \mathrm{H}_{7} \mathrm{~N}_{11} \mathrm{O}_{4}(297.20)$ : calcd C 24.25, H 2.37, N 51.84\%. Found: C 24.26, H 2.39, N 51.86\%.

\section{Computational Details}


Computations were performed by using the Gaussian 09 suite of programs. ${ }^{1}$ The elementary geometric optimization and the frequency analysis were performed at the level of the Becke three parameter, Lee-Yan-Parr (B3LYP) ${ }^{2}$ functional with the $6-311++\mathrm{G}^{* *}$ basis set. ${ }^{3}$ All of the optimized structures were characterized to be local energy minima on the potential surface without any imaginary frequencies. Atomization energies were calculated by the CBS-4M. ${ }^{4}$ All the optimized structures were characterized to be true local energy minima on the potential-energy surface without imaginary frequencies.

The predictions of heats of formation (HOF) of compounds used the hybrid DFTB3LYP methods with the 6-311++G** basis set through designed isodesmic reactions. The isodesmic reaction processes, that is, the number of each kind of formal bond is conserved, were used with the application of the bond separation reaction (BSR) rules. The molecule was broken down into a set of two heavy-atom molecules containing the same component bonds. The isodesmic reactions used to derive the HOF of compounds 2-8 are shown in Scheme S1.

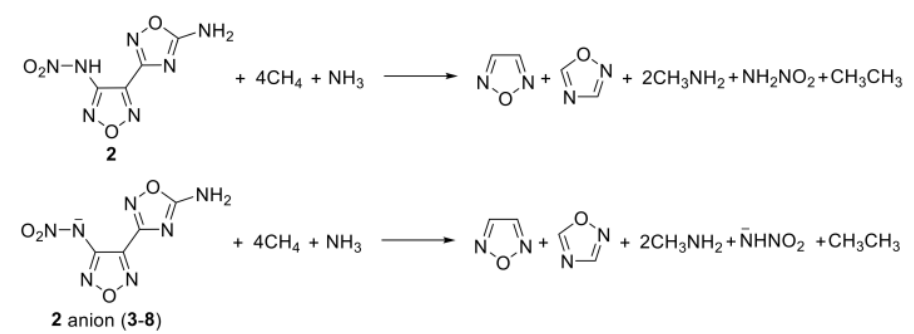

Scheme S1. Isodesmic reactions for calculating heats of formation for $\mathbf{2 - 8}$

For energetic salts, the solid-phase heat of formation is calculated based on a Born-Haber energy cycle (Scheme S2). ${ }^{5}$ The number is simplified by equation 1:

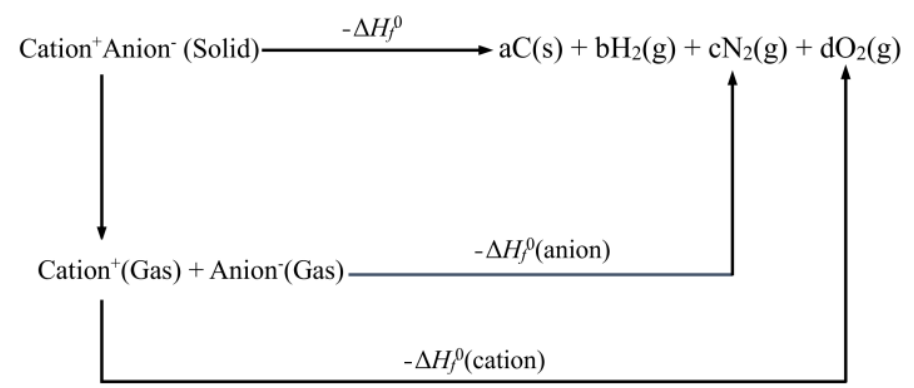

Scheme S2. Born-Haber Cycle for the formation of energetic salts.

$\Delta H_{\mathrm{f}}^{\mathrm{o}}($ salt, $298 \mathrm{~K})=\Delta H_{\mathrm{f}}^{\mathrm{o}}($ cation, $298 \mathrm{~K})+\Delta H_{\mathrm{f}}^{\mathrm{o}}($ anion, $298 \mathrm{~K})-\Delta H_{\mathrm{L}}(1)$

where $\Delta H_{\mathrm{L}}$ is the lattice energy of the salts, which could be predicted by using the formula suggested by Jenkins et al. [Eq. (2)]

$\Delta H_{\mathrm{L}}=\mathrm{U}_{\mathrm{POT}}+\left[p\left(\mathrm{n}_{\mathrm{M}} / 2-2\right)+q\left(\mathrm{n}_{\mathrm{X}} / 2-2\right)\right] R T(2)$

where $\mathrm{n}_{\mathrm{M}}$ and $\mathrm{n}_{\mathrm{X}}$ depend on the nature of the ions, $\mathrm{M}^{\mathrm{q}+}$ and $\mathrm{X}^{\mathrm{p}-}$, and are equal to 3 for monatomic ions, 5 for linear polyatomic ions, and 6 for nonlinear polyatomic ions.

The equation for lattice potential energy $U_{\text {РОT }}$ [Eq. (3)] has the form:

$U_{\text {РОт }}\left[\mathrm{kJ} \mathrm{mol}^{-1}\right]=\gamma\left(\rho_{\mathrm{m}} / \mathrm{M}_{\mathrm{m}}\right)^{1 / 3}+\delta(3)$

where $\rho_{\mathrm{m}}\left[\mathrm{g} \mathrm{cm}^{-3}\right]$ is the density of the salt, $\mathrm{M}_{\mathrm{m}}$ is the chemical formula mass of the ionic material, and values for (g) and the coefficients $\gamma(\mathrm{kJ}$ $\left.\mathrm{mol}^{-1} \mathrm{~cm}\right)$ and $\delta\left(\mathrm{kJ} \mathrm{mol}^{-1}\right)$ are assigned literature values. ${ }^{5}$

Table S1 Calculated zero-point energy (ZPE), thermal correction to enthalpy (HT), total energy (E0) and gas phase heats of formation (HOF)

\begin{tabular}{lllll}
\hline Compound & $\mathrm{ZPE} / \mathrm{au}$ & $\mathrm{H}_{\mathrm{T}} / \mathrm{au}$ & $\mathrm{E} / \mathrm{au}$ & $\mathrm{HOF}_{\mathrm{kJ}} \mathrm{mol}{ }^{-1}$ \\
\hline $\mathbf{2}$ & 0.105498 & 0.012241 & -838.1832304 & 300.4851298 \\
$\mathbf{2}$ anion & 0.093349 & 0.013105 & -837.6624871 & 271.466386 \\
$\mathrm{CH}_{4}$ & 0.043894 & 0.003821 & -40.5262 & -74.6 \\
$\mathrm{NH}_{3}$ & 0.033694 & 0.003825 & -56.5671081 & -45.9 \\
Fuzan & 0.044784 & 0.004503 & -262.0610572 & 196.75 \\
$1,2,4-$ oxadiazole & 0.045602 & 0.004451 & -262.101 & 99.39 \\
$\mathrm{CH}_{3} \mathrm{NH}_{2}$ & 0.062724 & 0.004419 & -95.8719773 & -23 \\
$\mathrm{NH}_{2} \mathrm{NO}_{2}$ & 0.037485 & 0.004404 & -261.0368506 & -6.11 \\
$\mathrm{CH}_{3} \mathrm{CH}_{3}$ & 0.073092 & 0.004484 & -79.8416782 & -84 \\
$\mathrm{~N}^{-} \mathrm{HNO}_{2}$ & 0.025624 & 0.004306 & -260.5046206 & -6.74 \\
\hline
\end{tabular}


The solid-state enthalpy of formation for neutral compound can be estimated by subtracting the heat of sublimation from gas-phase heat of formation. Based on the literature, ${ }^{6}$ the heat of sublimation can be estimated with Trouton's rule according to supplementary equation 1 , where $\mathrm{T}$ represents either the melting point or the decomposition temperature when no melting occurs prior to decomposition:

$$
\Delta H_{\text {sub }}=188 / \mathrm{J} \mathrm{mol}^{-1} \mathrm{~K}^{-1} \times \mathrm{T}
$$

Table S2 The decomposition temperatures $\left(T_{\mathrm{d}}\right)$, TBDEs and maximum values of ESP for 2-8

\begin{tabular}{llllllll}
\hline Compd. & $\mathbf{2}$ & $\mathbf{3}$ & $\mathbf{4}$ & $\mathbf{5}$ & $\mathbf{6}$ & $\mathbf{8}$ \\
\hline$T_{\mathrm{d}}$ & 111 & 206 & 192 & 175 & 235 & 190 \\
TBDE & 186.86 & 368.09 & 367.42 & 366.41 & 369.91 & 367.08 \\
maximum value of ESP & 63.83 & 42.24 & 58.43 & 60.53 & 43.82 & 59.32 & 59.28 \\
\hline
\end{tabular}

\section{Crystallographic data for $2-8$}

Table S3 Crystallographic data of 2-8.

\begin{tabular}{|c|c|c|c|c|c|c|c|}
\hline Compd. & 2 & 3 & 4 & 5 & 6 & 7 & 8 \\
\hline CCDC number & 1938981 & 1938982 & 1938983 & 1938984 & 1938987 & 1938985 & 1938986 \\
\hline formula & $\mathrm{C}_{4} \mathrm{H}_{3} \mathrm{~N}_{7} \mathrm{O}_{4}$ & $\mathrm{C}_{4} \mathrm{H}_{10} \mathrm{~N}_{8} \mathrm{O}_{6}$ & $\mathrm{C}_{4} \mathrm{H}_{7} \mathrm{~N}_{9} \mathrm{O}_{4}$ & $\mathrm{C}_{4} \mathrm{H}_{8} \mathrm{~N}_{8} \mathrm{O}_{6}$ & $\mathrm{C}_{10} \mathrm{H}_{18} \mathrm{~N}_{20} \mathrm{O}_{9}$ & $\mathrm{C}_{5} \mathrm{H}_{9} \mathrm{~N}_{11} \mathrm{O}_{5}$ & $\mathrm{C}_{6} \mathrm{H}_{9} \mathrm{~N}_{11} \mathrm{O}_{5}$ \\
\hline crystal system & Monoclinic & Triclinic & Monoclinic & Triclinic & Monoclinic & Tric linic & Monoclinic \\
\hline space group & $P 2{ }_{1} / \mathrm{c}$ & $P-1$ & $P 21 / \mathrm{n}$ & $P-1$ & $C 2 / \mathrm{c}$ & $P 1$ & $P 21 / \mathrm{n}$ \\
\hline $\mathrm{a}[\AA]$ & $5.2562(2)$ & $6.6872(5)$ & $3.7442(2)$ & $6.7659(3)$ & $36.197(5)$ & $4.9731(4)$ & $5.0822(3)$ \\
\hline $\mathrm{b}[\AA]$ & $16.8557(8)$ & $8.3323(5)$ & $15.6661(7)$ & $8.2228(4)$ & $3.5810(5)$ & $7.1624(7)$ & $20.5085(11)$ \\
\hline c $[\AA]]$ & $8.8720(4)$ & $9.8903(6)$ & $15.5963(7)$ & $9.8059(5)$ & $17.064(2)$ & $8.6086(8)$ & $11.9101(7)$ \\
\hline$\alpha\left[^{\circ}\right]$ & 90 & $88.604(2)$ & 90 & $86.4560(10)$ & 90 & $79.379(4)$ & 90 \\
\hline$\beta\left[^{\circ}\right]$ & $98.242(2)$ & $80.093(2)$ & $91.876(3)$ & $69.944(2)$ & $96.208(7)$ & $79.326(3)$ & $92.412(2)$ \\
\hline$\gamma\left[{ }^{\circ}\right]$ & 90 & $79.909(2)$ & 90 & $82.1400(10)$ & 90 & $70.938(3)$ & 90 \\
\hline $\mathrm{V}\left[\AA^{3}\right]$ & $777.91(6)$ & $534.46(6)$ & $914.34(8)$ & $507.58(4)$ & $2198.9(5)$ & $282.26(4)$ & $1240.27(12)$ \\
\hline Z & 4 & 2 & 4 & 2 & 4 & 1 & 4 \\
\hline$\lambda[\AA]$ & 0.71073 & 0.71073 & 0.71073 & 0.71073 & 0.71073 & 0.71073 & 0.71073 \\
\hline$\rho_{\text {calcd }}\left[\mathrm{g} \mathrm{cm}^{-3}\right]$ & 1.820 & 1.654 & 1.781 & 1.729 & 1.699 & 1.784 & 1.688 \\
\hline$\mu\left[\mathrm{mm}^{-1}\right]$ & 0.162 & 0.151 & 0.156 & 0.159 & 0.147 & 0.156 & 0.146 \\
\hline$F(000)$ & 432 & 276 & 504 & 272 & 1160 & 156 & 648 \\
\hline$\theta$ range $\left[{ }^{\circ}\right]$ & $2.417-27.511$ & $3.140-27.534$ & $2.600-27.478$ & $3.230-27.557$ & $1.132-27.569$ & $2.429-27.567$ & $2.622-27.553$ \\
\hline Data/restraints/parameter & $1772 / 0 / 136$ & $2438 / 0 / 175$ & $2079 / 0 / 155$ & $3866 / 3 / 174$ & $2544 / 0 / 177$ & $2369 / 3 / 191$ & $2857 / 0 / 200$ \\
\hline S & 1.048 & 1.041 & 1.036 & 1.051 & 1.024 & 1.059 & 1.063 \\
\hline $\mathrm{R}_{1}[I>2 \sigma(I)]$ & $0.0382(1384)$ & $0.0372(2000)$ & $0.0700(1156)$ & $0.0394(3307)$ & $0.0528(1526)$ & $0.0312(2245)$ & $0.0397(2114)$ \\
\hline $\mathrm{wR}_{2}[I>2 \sigma(I)]$ & $0.1075(1772)$ & $0.1046(2438)$ & $0.1342(2079)$ & $0.1063(3866)$ & $0.1367(2544)$ & $0.0813(2369)$ & $0.1088(2857)$ \\
\hline
\end{tabular}

Table S4 Selected bond lengths $[\AA]$, bond angles $\left[{ }^{\circ}\right]$ and dihedral angles $\left[{ }^{\circ}\right]$ for compound 2

\begin{tabular}{|l|l|l|l|}
\hline C1-N2 & $1.3009(17)$ & N6-C3-C2 & $119.07(12)$ \\
C1-N3 & $1.3775(16)$ & N5-C3-C2 & $123.66(11)$ \\
C1-C2 & $1.4232(17)$ & N7-C4-N5 & $128.60(12)$ \\
C2-N1 & $1.2920(16)$ & N7-C4-O4 & $118.37(11)$ \\
C2-C3 & $1.4595(17)$ & N5-C4-O4 & $113.03(11)$ \\
C3-N6 & $1.2942(16)$ & C2-N1-O1 & $105.88(11)$ \\
C3-N5 & $1.3568(16)$ & C1-N2-O1 & $104.29(10)$ \\
C4-N7 & $1.3100(16)$ & N4-N3-C1 & $124.42(12)$ \\
C4-N5 & $1.3188(16)$ & N4-N3-H3 & 117.8 \\
C4-O4 & $1.3514(16)$ & C1-N3-H3 & 117.8 \\
N1-O1 & $1.3636(15)$ & O2-N4-O3 & $126.44(13)$ \\
N2-O1 & $1.3964(15)$ & O2-N4-N3 & $118.00(12)$ \\
N3-N4 & $1.3562(16)$ & O3-N4-N3 & $115.55(12)$ \\
N3-H3 & 0.8600 & C4-N5-C3 & $101.33(10)$ \\
N4-O2 & $1.2107(15)$ & C3-N6-O4 & $102.37(10)$ \\
N4-O3 & $1.2170(16)$ & C4-N7-H7A & 120.0 \\
N6-O4 & $1.4194(13)$ & H7A-N7-H7B & 120.0 \\
N7-H7A & 0.8600 & N1-O1-N2 & 120.0 \\
N7-H7B & 0.8600 & C4-O4-N6 & $111.30(10)$ \\
N2-C1-N3 & $127.55(12)$ & N1-C2-C3 & $106.01(9)$ \\
N2-C1-C2 & $109.46(11)$ & O2-N4-N3-C1 & $122.96(12)$ \\
N3-C1-C2 & $122.99(11)$ & N2-C1-C2-C3 & $-0.885(191)$ \\
N1-C2-C1 & $109.08(11)$ & N1-C2-C3-N5 & $-179.59(13)$ \\
C1-C2-C3 & $127.96(11)$ & N6-O4-C4-N7 & $-1.0(2)$ \\
N6-C3-N5 & $117.26(11)$ & & $-179.642(105)$ \\
\hline
\end{tabular}


Table S5 Hydrogen bonds present in compound 2.

\begin{tabular}{lllll}
\hline $\mathrm{D}-\mathrm{H} \cdots \mathrm{A}$ & $\mathrm{D}-\mathrm{H} / \AA$ & $\mathrm{H} \cdots \mathrm{A} / \AA$ & $\mathrm{D} \cdots \mathrm{A} / \AA$ & $\mathrm{D}-\mathrm{H} \cdots \mathrm{A} /{ }^{\circ}$ \\
\hline $\mathrm{N} 7-\mathrm{H} 7 \mathrm{~B} \cdots \mathrm{O} 2$ & 0.86 & 2.44 & $3.1497(16)$ & 140.0 \\
$\mathrm{~N} 7-\mathrm{H} 7 \mathrm{~B} \cdots \mathrm{N} 2$ & 0.86 & 2.36 & $3.1473(17)$ & 152.5 \\
$\mathrm{~N} 7-\mathrm{H} 7 \mathrm{~A} \cdots \mathrm{N} 5$ & 0.86 & 2.19 & $3.0093(16)$ & 158.6 \\
$\mathrm{~N} 3-\mathrm{H} 3 \cdots \mathrm{N} 6$ & 0.86 & 2.19 & $2.8274(16)$ & 130.6 \\
\hline
\end{tabular}

Table S6 Selected bond lengths $[\AA]$, bond angles $\left[{ }^{\circ}\right]$ and dihedral angles $\left[^{\circ}\right]$ for compound $\mathbf{3}$.

\begin{tabular}{|l|l|l|l|}
\hline C1-N1 & $1.3108(16)$ & N3-C3-N4 & $116.28(11)$ \\
C1-N5 & $1.3776(16)$ & N3-C3-C2 & $122.52(11)$ \\
C1-C2 & $1.4387(16)$ & N4-C3-C2 & $121.20(11)$ \\
C2-N2 & $1.2959(17)$ & N4-C4-N7 & $127.95(12)$ \\
C2-C3 & $1.4621(16)$ & N4-C4-O2 & $113.06(10)$ \\
C3-N3 & $1.3000(17)$ & N7-C4-O2 & $118.98(11)$ \\
C3-N4 & $1.3653(15)$ & C1-N1-O1 & $105.08(10)$ \\
C4-N4 & $1.3133(16)$ & C2-N2-O1 & $105.65(10)$ \\
C4-N7 & $1.3178(16)$ & C3-N3-O2 & $102.43(9)$ \\
C4-O2 & $1.3463(15)$ & C4-N4-C3 & $102.08(10)$ \\
N1-O1 & $1.3937(15)$ & N6-N5-C1 & $117.04(10)$ \\
N2-O1 & $1.3669(15)$ & O4-N6-O3 & $120.79(10)$ \\
N3-O2 & $1.4288(13)$ & O4-N6-N5 & $123.85(11)$ \\
N5-N6 & $1.3233(14)$ & O3-N6-N5 & $115.36(11)$ \\
N6-O4 & $1.2440(14)$ & C4-N7-H7A & 118.6 \\
N6-O3 & $1.2496(15)$ & C4-N7-H7B & 122.5 \\
N7-H7A & 0.9108 & H7A-N7-H7B & 118.8 \\
N7-H7B & 0.8487 & N2-O1-N1 & $111.51(9)$ \\
N8-H8A & $0.978(18)$ & C4-O2-N3 & $106.14(9)$ \\
N8-H8B & $0.858(19)$ & H8A-N8-H8B & $114.1(14)$ \\
N8-H8C & $0.905(19)$ & H8A-N8-H8C & $105.2(14)$ \\
N8-H8D & $0.848(19)$ & H8B-N8-H8C & $111.7(16)$ \\
O5-H5A & 0.9568 & H8A-N8-H8D & $111.5(15)$ \\
O5-H5B & 0.9159 & H8B-N8-H8D & $108.6(16)$ \\
O6-H6B & 0.8965 & H8C-N8-H8D & $105.4(16)$ \\
O6-H6A & 0.9368 & H5A-O5-H5B & 105.6 \\
N1-C1-N5 & $131.13(11)$ & H6B-O6-H6A & 108.7 \\
N1-C1-C2 & $108.16(11)$ & C3-N4-C4-N7 & $179.053(130)$ \\
N5-C1-C2 & $120.70(11)$ & O4-N6-N5-C1 & $2.242(183)$ \\
N2-C2-C1 & $109.60(11)$ & C2-C3-N4-C4 & $-179.22(11)$ \\
N2-C2-C3 & $120.13(11)$ & O1-N2-C2-C3 & $-179.179(107)$ \\
C1-C2-C3 & $130.27(11)$ & & \\
\hline
\end{tabular}

Table S7 Hydrogen bonds present in compound $\mathbf{3}$.

\begin{tabular}{lllll}
\hline $\mathrm{D}-\mathrm{H} \cdots \mathrm{A}$ & $\mathrm{D}-\mathrm{H} / \AA$ & $\mathrm{H} \cdots \mathrm{A} / \AA$ & $\mathrm{D} \cdots \mathrm{A} / \AA$ & $\mathrm{D}-\mathrm{H} \cdots \mathrm{A} /{ }^{\circ}$ \\
\hline $\mathrm{N} 7-\mathrm{H} 7 \mathrm{~A} \cdots \mathrm{O} 5$ & 0.91 & 2.05 & $2.9334(16)$ & 164.1 \\
$\mathrm{~N} 7-\mathrm{H} 7 \mathrm{~B} \cdots \mathrm{N} 1$ & 0.85 & 2.32 & $2.9285(15)$ & 129.4 \\
$\mathrm{~N} 7-\mathrm{H} 7 \mathrm{~B} \cdots \mathrm{O} 4$ & 0.85 & 2.20 & $3.0134(16)$ & 160.8 \\
$\mathrm{~N} 8-\mathrm{H} 8 \mathrm{~A} \cdots \mathrm{O} 6$ & $0.978(18)$ & $1.892(18)$ & $2.8573(17)$ & $168.7(14)$ \\
$\mathrm{N} 8-\mathrm{H} 8 \mathrm{~B} \cdots \mathrm{O} 4$ & $0.858(19)$ & $2.059(19)$ & $2.9138(16)$ & $174.5(15)$ \\
$\mathrm{N} 8-\mathrm{H} 8 \mathrm{C} \cdots \mathrm{O} 6$ & $0.905(19)$ & $2.020(19)$ & $2.9005(18)$ & $164.1(15)$ \\
$\mathrm{N} 8-\mathrm{H} 8 \mathrm{D} \cdots \mathrm{N} 3$ & $0.848(19)$ & $2.673(17)$ & $3.1118(17)$ & $113.7(13)$ \\
$\mathrm{N} 8-\mathrm{H} 8 \mathrm{D} \cdots \mathrm{N} 5$ & $0.848(19)$ & $2.23(2)$ & $3.0673(17)$ & $170.3(16)$ \\
\hline
\end{tabular}

Table S8 Selected bond lengths $[\AA]$, bond angles $\left[{ }^{\circ}\right]$ and dihedral angles $\left[{ }^{\circ}\right]$ for compound 4.

\begin{tabular}{|l|l|l|l|}
\hline C1-N1 & $1.313(3)$ & N3-C3-C2 & $120.3(2)$ \\
C1-N7 & $1.317(3)$ & C4-C3-C2 & $129.6(2)$ \\
C1-O1 & $1.342(3)$ & N4-C4-N5 & $131.3(2)$ \\
C2-N2 & $1.298(3)$ & N4-C4-C3 & $108.3(2)$ \\
C2-N1 & $1.366(3)$ & C1-N1-C2 & $120.4(2)$ \\
C2-C3 & $1.458(4)$ & C2-N2-O1 & $101.7(2)$ \\
C3-N3 & $1.295(3)$ & C3-N3-O2 & $102.69(19)$ \\
C3-C4 & $1.434(4)$ & C4-N4-O2 & $105.3(2)$ \\
C4-N4 & $1.320(3)$ & N6-N5-C4 & $104.7(2)$ \\
C4-N5 & $1.373(3)$ & O3-N6-O4 & $116.8(2)$ \\
N2-O1 & $1.423(3)$ & O3-N6-N5 & $121.1(2)$ \\
N3-O2 & $1.378(3)$ & O4-N6-N5 & $123.3(2)$ \\
N4-O2 & $1.395(3)$ & C1-N7-H7A & $115.6(2)$ \\
N5-N6 & $1.319(3)$ & H7A-N7-H7B & 120.0 \\
N6-O3 & $1.236(3)$ & C1-O1-N2 & 120.0 \\
N6-O4 & $1.266(3)$ & N3-O2-N4 & 120.0 \\
N7-H7A & 0.8600 & N9-N8-H8A & $105.96(18)$ \\
N7-H7B & 0.8600 & N9-N8-H8B & $111.59(18)$ \\
N8-N9 & $1.440(3)$ & H8A-N8-H8B & 109.5 \\
N8-H8A & 0.8900 & N9-N8-H8C & 109.5 \\
N8-H8B & 0.8900 & H8A-N8-H8C & 109.5 \\
N8-H8C & 0.8900 & H8B-N8-H8C & 109.5 \\
N9-H9A & 0.8900 & N8-N9-H9A & 109.5 \\
N9-H9B & 0.8900 & N8-N9-H9B & 109.5 \\
N1-C1-N7 & $129.1(3)$ & H(9A)-N(9)-H(9B) & 109.1 \\
N1-C1-O1 & $113.5(2)$ & O3-N6-N5-N4 & 109.2 \\
N7-C1-O1 & $117.4(2)$ & N4-C4-C3-C2 & 109.5 \\
N2-C2-N1 & $116.2(2)$ & & $0.059(366)$ \\
N2-C2-C3 & $121.6(2)$ & $178.702(257)$ \\
\hline
\end{tabular}




\begin{tabular}{|l|l|l|l|}
\hline N1-C2-C3 & $122.3(2)$ & C3-C2-N1-C1 & $178.4(2)$ \\
N3-C3-C4 & $110.1(2)$ & C2-N1-C1-N7 & $179.333(278)$ \\
\hline
\end{tabular}

Table S9 Hydrogen bonds present in compound 4 .

\begin{tabular}{lllll}
\hline $\mathrm{D}-\mathrm{H} \cdots \mathrm{A}$ & $\mathrm{D}-\mathrm{H} / \AA$ & $\mathrm{H} \cdots \mathrm{A} / \AA$ & $\mathrm{D} \cdots \mathrm{A} / \AA$ & $\mathrm{D}-\mathrm{H} \cdots \mathrm{A} /{ }^{\circ}$ \\
\hline $\mathrm{N} 7-\mathrm{H} 7 \mathrm{~A} \cdots \mathrm{O} 4$ & 0.86 & 2.01 & $2.825(3)$ & 158.8 \\
$\mathrm{~N} 7-\mathrm{H} 7 \mathrm{~B} \cdots \mathrm{O} 3$ & 0.86 & 2.09 & $2.864(3)$ & 149.4 \\
$\mathrm{~N} 8-\mathrm{H} 8 \mathrm{~A} \cdots \mathrm{N} 2$ & 0.89 & 2.45 & $2.943(3)$ & 115.0 \\
$\mathrm{~N} 8-\mathrm{H} 8 \mathrm{~A} \cdots \mathrm{N} 5$ & 0.89 & 2.13 & $2.999(3)$ & 163.7 \\
\hline
\end{tabular}

Table S10 Selected bond lengths $[\AA ̊]$, bond angles $\left[{ }^{\circ}\right]$ and dihedral angles $\left[{ }^{\circ}\right]$ for compound $\mathbf{5}$.

\begin{tabular}{|l|l|l|l|}
\hline C1-N1 & $1.313(2)$ & N3-C1-C2 & $120.83(13)$ \\
C1-N3 & $1.3742(19)$ & N2-C2-C1 & $109.46(13)$ \\
C1-C2 & $1.4397(19)$ & N2-C2-C3 & $119.85(13)$ \\
C2-N2 & $1.296(2)$ & C1-C2-C3 & $130.69(14)$ \\
C2-C3 & $1.4585(19)$ & N5-C3-N6 & $116.07(13)$ \\
C3-N5 & $1.299(2)$ & N5-C3-C2 & $122.56(13)$ \\
C3-N6 & $1.3641(18)$ & N6-C3-C2 & $121.35(13)$ \\
C4-N7 & $1.3130(19)$ & N7-C4-N6 & $128.35(15)$ \\
C4-N6 & $1.3231(19)$ & N7-C4-O4 & $118.75(14)$ \\
C4-O4 & $1.3421(19)$ & N6-C4-O4 & $112.90(13)$ \\
N1-O1 & $1.3953(17)$ & C1-N1-O1 & $105.33(12)$ \\
N2-O1 & $1.3672(16)$ & C2-N2-O1 & $106.05(12)$ \\
N3-N4 & $1.3200(17)$ & N4-N3-C1 & $116.13(12)$ \\
N4-O2 & $1.2418(18)$ & O2-N4-O3 & $119.92(13)$ \\
N4-O3 & $1.2650(18)$ & O2-N4-N3 & $124.80(13)$ \\
N5-O4 & $1.4243(16)$ & O3-N4-N3 & $115.28(13)$ \\
N7-H7A & 0.8600 & C3-N5-O4 & $102.87(11)$ \\
N7-H7B & C4-N6-C3 & $101.96(13)$ \\
N8-O6 & 0.8600 & C4-N7-H7A & 120.0 \\
N8-H8A & $1.3965(19)$ & C4-N7-H7B & 120.0 \\
N8-H8B & 0.8900 & H7A-N7-H7B & 120.0 \\
N8-H8C & 0.8900 & O6-N8-H8A & 109.5 \\
O5-H5A & 0.8900 & O6-N8-H8B & 109.5 \\
O5-H5B & $0.85(2)$ & H8A-N8-H8B & 109.5 \\
O6-H6 & $0.837(19)$ & O6-N8-H8C & 109.5 \\
N2-O1-N1 & 0.8200 & H8A-N8-H8C & 109.5 \\
C4-O4-N5 & $111.13(11)$ & H8B-N8-H8C & 109.5 \\
H5A-O5-H5B & O2-N4-N3-C1 & $-2.180(216)$ \\
N8-O6-H6 & N3-C1-C2-N2 & $178.354(134)$ \\
N1-C1-N3 & N2-C1-C3-N5 & $177.344(145)$ \\
N1-C1-C2 & C3-N6-C4-N7 & $-179.532(161)$ \\
\hline
\end{tabular}

Table S11 Hydrogen bonds present in compound 5.

\begin{tabular}{lllll}
\hline $\mathrm{D}-\mathrm{H} \cdots \mathrm{A}$ & $\mathrm{D}-\mathrm{H} / \AA$ & $\mathrm{H} \cdots \mathrm{A} / \AA$ & $\mathrm{D} \cdots \mathrm{A} / \AA$ & $\mathrm{D}-\mathrm{H}^{\cdots} \mathrm{A} /{ }^{\circ}$ \\
\hline $\mathrm{O} 6-\mathrm{H} 6 \cdots \mathrm{N} 6$ & 0.82 & 1.93 & $2.7481(19)$ & 172.7 \\
$\mathrm{O} 5-\mathrm{H} 5 \mathrm{~B} \cdots \mathrm{O} 6$ & $0.837(19)$ & $2.44(2)$ & $3.063(2)$ & $132(2)$ \\
$\mathrm{O} 5-\mathrm{H} 5 \mathrm{~A} \cdots \mathrm{O} 3$ & $0.85(2)$ & $2.14(2)$ & $2.986(2)$ & $178(4)$ \\
$\mathrm{O} 5-\mathrm{H} 5 \mathrm{~A} \cdots \mathrm{O} 2$ & $0.85(2)$ & $2.67(3)$ & $3.267(2)$ & $129(3)$ \\
$\mathrm{N} 8-\mathrm{H} 8 \mathrm{C} \cdots \mathrm{N} 5$ & 0.89 & 2.09 & $2.9274(19)$ & 157.3 \\
$\mathrm{~N} 8-\mathrm{H} 8 \mathrm{C} \cdots \mathrm{N} 3$ & 0.89 & 2.34 & $2.8785(19)$ & 118.8 \\
$\mathrm{~N} 8-\mathrm{H} 8 \mathrm{~B} \cdots \mathrm{O} 3$ & 0.89 & 2.15 & $3.017(2)$ & 165.3 \\
$\mathrm{~N} 8-\mathrm{H} 8 \mathrm{~A} \cdots \mathrm{O} 5$ & 0.89 & 2.10 & $2.949(3)$ & 158.5 \\
$\mathrm{~N} 7-\mathrm{H} 7 \mathrm{~B} \cdots \mathrm{O} 2$ & 0.86 & 2.23 & $3.056(2)$ & 161.3 \\
$\mathrm{~N} 7-\mathrm{H} 7 \mathrm{~B} \cdots \mathrm{N} 1$ & 0.86 & 2.24 & $2.8631(18)$ & 128.8 \\
$\mathrm{~N} 7-\mathrm{H} 7 \mathrm{~A} \cdots \mathrm{O} 5$ & 0.86 & 2.09 & $2.933(2)$ & 165.2 \\
\hline
\end{tabular}

Table S12 Selected bond lengths $[\AA]$, bond angles $\left[{ }^{\circ}\right]$ and dihedral angles $\left[^{\circ}\right]$ for compound $\mathbf{6}$.

\begin{tabular}{|l|l|l|l|}
\hline C1-N1 & $1.311(3)$ & C4-C3-C2 & $131.3(2)$ \\
C1-N2 & $1.317(3)$ & N5-C4-N6 & $131.5(2)$ \\
C1-O1 & $1.346(3)$ & N5-C4-C3 & $108.0(2)$ \\
C2-N3 & N6-C4-C3 & $120.5(2)$ \\
C2-N2 & N10-C5-N8 & $120.2(2)$ \\
C2-C3 & $1.362(3)$ & N10-C5-N9 & $120.1(2)$ \\
C3-N4 & $1.476(3)$ & N8-C5-N9 & $119.7(2)$ \\
C3-C4 & $1.298(3)$ & C1-N1-H1A & 120.0 \\
C4-N5 & $1.433(3)$ & C1-N1-H1B & 120.0 \\
C4-N6 & $1.309(3)$ & H1A-N1-H1B & 120.0 \\
C5-N10 & $1.373(3)$ & C2-N2-C2 & $102.24(18)$ \\
C5-N8 & $1.312(3)$ & C3-N4-O1 & $102.49(16)$ \\
C5-N9 & C4-N5-O2 & $105.51(18)$ \\
N1-H1A & $1.313(3)$ & N7-N6-C4 & $105.54(18)$ \\
N1-H1B & $1.318(3)$ & O4-N7-O3 & $116.12(19)$ \\
N3-O1 & 0.8600 & O4-N7-N6 & $119.70(19)$ \\
N4-O2 & 0.8600 & O3-N7-N6 & $117.1(2)$ \\
N5-O2 & $1.433(2)$ & C5-N10-H10A & $123.2(2)$ \\
N6-N7 & $1.375(2)$ & C5-N10-H10B & 120.0 \\
N7-O4 & $1.404(3)$ & H10A-N10-H10B & 120.0 \\
N7-O3 & $1.316(3)$ & C5-N8-H8A & 120.0 \\
N10-H10A & $1.250(2)$ & C5-N8-H8B & 120.0 \\
N10-H10B & $1.256(2)$ & & 120.0 \\
\hline
\end{tabular}




\begin{tabular}{|l|l|l|l|}
\hline N8-H8A & 0.8600 & H8A-N8-H8B & 120.0 \\
N8-H8B & 0.8600 & C5-N9-H9A & 120.0 \\
N9-H9A & 0.8600 & C5-N9-H9B & 120.0 \\
N9-H9B & 0.8600 & H9A-N9-H9B & 120.0 \\
O5-H5A & 0.8816 & N4-O1-N3 & $106.07(16)$ \\
O5-H5B & 0.8514 & H5A-O5-H5B & $110.68(16)$ \\
O5-H5A & 0.8815 & H5A-O5-H5A & 106.7 \\
O5-H5B & 0.8516 & H5B-O5-H5A & 98.8 \\
N1-C1-N2 & $128.2(2)$ & H5A-O5-H5B & 85.9 \\
N1-C1-O1 & $119.1(2)$ & H5B-O5-H5B & 85.8 \\
N2-C1-O1 & $112.71(19)$ & H1-C1-N2-C2 & 161.0 \\
N3-C2-N2 & $116.49(19)$ & N2-C2-C3-N4 & 106.7 \\
N3-C2-C3 & $123.25(19)$ & C3-C4-N6-N7 & $179.8(2)$ \\
N2-C2-C3 & $120.25(19)$ & O2-N5-C4-N6 & $-13.5(3)$ \\
N4-C3-C4 & $110.2(2)$ & $-173.59(19)$ \\
N4-C3-C2 & $118.53(19)$ & & $177.654(224)$ \\
\hline
\end{tabular}

Table S13 Hydrogen bonds present in compound 6.

\begin{tabular}{|c|c|c|c|c|}
\hline D-H $\cdots A$ & D-H/Å & $\mathrm{H} \cdots \mathrm{A} / \AA$ & $\mathrm{D} \cdots \mathrm{A} / \AA$ & $\mathrm{D}-\mathrm{H} \cdots \mathrm{A} /{ }^{\circ}$ \\
\hline N1-H1A...N2 & 0.86 & 2.15 & $2.979(3)$ & 162.8 \\
\hline N1-H1B...O5 & 0.86 & 2.24 & $3.006(2)$ & 147.9 \\
\hline N10-H10A...N3 & 0.86 & 2.67 & $3.410(3)$ & 144.3 \\
\hline N10-H10A...N6 & 0.86 & 2.41 & $3.107(3)$ & 139.2 \\
\hline N10-H10B...O4 & 0.86 & 2.29 & $3.074(3)$ & 152.5 \\
\hline N10-H10B...O4 & 0.86 & 2.42 & $2.914(3)$ & 117.1 \\
\hline N8-H8A...N3 & 0.86 & 2.40 & $3.198(3)$ & 154.9 \\
\hline N8-H8A...O1 & 0.86 & 2.44 & $3.238(3)$ & 153.6 \\
\hline N8-H8B...N5 & 0.86 & 2.26 & $3.058(3)$ & 153.5 \\
\hline N8-H8B...O3 & 0.86 & 2.52 & $3.240(3)$ & 142.5 \\
\hline N9-H9A...O3 & 0.86 & 2.27 & $3.081(3)$ & 157.1 \\
\hline N9-H9A...O4 & 0.86 & 2.47 & $3.216(3)$ & 145.0 \\
\hline N9-H9B...O3 & 0.86 & 2.12 & $2.944(3)$ & 159.9 \\
\hline O5-H5A...N4 & 0.88 & 2.23 & $3.068(2)$ & 159.0 \\
\hline O5-H5B...N1 & 0.85 & 2.39 & $3.006(2)$ & 129.3 \\
\hline
\end{tabular}

Table S14 Selected bond lengths $[\AA]$, bond angles $\left[{ }^{\circ}\right]$ and dihedral angles $\left[{ }^{\circ}\right]$ for compound 7.

\begin{tabular}{|c|c|c|c|}
\hline C1-N7 & $1.311(3)$ & $\mathrm{N} 1-\mathrm{C} 1-\mathrm{O} 1$ & $113.2(2)$ \\
\hline $\mathrm{C} 1-\mathrm{N} 1$ & $1.315(3)$ & $\mathrm{N} 2-\mathrm{C} 2-\mathrm{N} 1$ & $116.3(2)$ \\
\hline $\mathrm{C} 1-\mathrm{O} 1$ & $1.348(3)$ & $\mathrm{N} 2-\mathrm{C} 2-\mathrm{C} 3$ & $122.2(2)$ \\
\hline $\mathrm{C} 2-\mathrm{N} 2$ & $1.303(3)$ & $\mathrm{N} 1-\mathrm{C} 2-\mathrm{C} 3$ & $121.5(2)$ \\
\hline $\mathrm{C} 2-\mathrm{N} 1$ & $1.365(3)$ & $\mathrm{N} 3-\mathrm{C} 3-\mathrm{C} 4$ & $109.4(2)$ \\
\hline $\mathrm{C} 2-\mathrm{C} 3$ & $1.460(3)$ & $\mathrm{N} 3-\mathrm{C} 3-\mathrm{C} 2$ & $119.7(2)$ \\
\hline $\mathrm{C} 3-\mathrm{N} 3$ & $1.300(3)$ & $\mathrm{C} 4-\mathrm{C} 3-\mathrm{C} 2$ & $130.8(2)$ \\
\hline $\mathrm{C} 3-\mathrm{C} 4$ & $1.436(3)$ & N4-C4-N5 & $130.9(2)$ \\
\hline C4-N4 & $1.313(3)$ & $\mathrm{N} 4-\mathrm{C} 4-\mathrm{C} 3$ & $108.5(2)$ \\
\hline C4-N5 & $1.379(3)$ & $\mathrm{N} 5-\mathrm{C} 4-\mathrm{C} 3$ & $120.5(2)$ \\
\hline $\mathrm{C} 5-\mathrm{O} 5$ & $1.223(3)$ & $\mathrm{O} 5-\mathrm{C} 5-\mathrm{N} 9$ & $124.4(2)$ \\
\hline C5-N9 & $1.330(3)$ & O5-C5-N10 & $121.0(2)$ \\
\hline $\mathrm{C} 5-\mathrm{N} 10$ & $1.401(3)$ & N9-C5-N10 & $114.6(2)$ \\
\hline N2-O1 & $1.425(3)$ & C1-N1-C2 & $101.9(2)$ \\
\hline $\mathrm{N} 3-\mathrm{O} 2$ & $1.371(3)$ & $\mathrm{C} 2-\mathrm{N} 2-\mathrm{O} 1$ & $102.54(19)$ \\
\hline $\mathrm{N} 4-\mathrm{O} 2$ & $1.393(3)$ & $\mathrm{C} 3-\mathrm{N} 3-\mathrm{O} 2$ & $105.6(2)$ \\
\hline N5-N6 & $1.306(3)$ & $\mathrm{C} 4-\mathrm{N} 4-\mathrm{O} 2$ & $104.9(2)$ \\
\hline N6-O4 & $1.245(3)$ & $\mathrm{N} 6-\mathrm{N} 5-\mathrm{C} 4$ & $117.0(2)$ \\
\hline N6-O3 & $1.265(3)$ & O4-N6-O3 & $119.5(2)$ \\
\hline N7-H7A & 0.8600 & O4-N6-N5 & $124.6(2)$ \\
\hline N7-H7B & 0.8600 & O3-N6-N5 & $115.9(2)$ \\
\hline N8-N9 & $1.395(4)$ & $\mathrm{C} 1-\mathrm{N} 7-\mathrm{H} 7 \mathrm{~A}$ & 120.0 \\
\hline N8-H8A & 0.8900 & C1-N7-H7B & 120.0 \\
\hline N8-H8B & 0.8900 & H7A-N7-H7B & 120.0 \\
\hline N9-H9 & 0.8600 & N9-N8-H8A & 109.3 \\
\hline N10-N11 & $1.426(3)$ & N9-N8-H8B & 109.3 \\
\hline N10-H10 & 0.8600 & H8A-N8-H8B & 109.5 \\
\hline N11-H11A & 0.8900 & C5-N9-N8 & $121.4(2)$ \\
\hline N11-H11B & 0.8900 & C5-N9-H9 & 119.3 \\
\hline N11-H11C & 0.8900 & N8-N9-H9 & 119.3 \\
\hline N10-N11-H11B & 109.5 & $\mathrm{C} 5-\mathrm{N} 10-\mathrm{N} 11$ & $111.65(19)$ \\
\hline H11A-N11-H11B & 109.5 & C5-N10-H10 & 124.2 \\
\hline N10-N11-H11C & 109.5 & N11-N10-H10 & 124.2 \\
\hline H11A-N11-H11C & 109.5 & N10-N11-H11A & 109.5 \\
\hline H11B-N11-H11C & 109.5 & O4-N6-N5-N4 & $-1.410(363)$ \\
\hline C1-O1-N2 & $106.11(18)$ & $\mathrm{N} 4-\mathrm{C} 4-\mathrm{C} 3-\mathrm{C} 2$ & $-178.564(269)$ \\
\hline $\mathrm{N} 3-\mathrm{O} 2-\mathrm{N} 4$ & $111.56(19)$ & $\mathrm{N} 2-\mathrm{C} 2-\mathrm{N} 1-\mathrm{C} 1$ & $0.1(3)$ \\
\hline N7-C1-N1 & $128.3(2)$ & $\mathrm{N} 1-\mathrm{C} 1-\mathrm{O} 1-\mathrm{N} 7$ & $-179.894(405)$ \\
\hline N7-C1-O1 & $118.6(2)$ & & \\
\hline
\end{tabular}

Table S15 Hydrogen bonds present in compound 7.

\begin{tabular}{|c|c|c|c|c|}
\hline $\mathrm{D}-\mathrm{H} \cdots \mathrm{A}$ & $\mathrm{D}-\mathrm{H} / \AA$ & $\mathrm{H} \cdots \mathrm{A} / \AA$ & $\mathrm{D} \cdots \mathrm{A} / \AA ̊$ & $\mathrm{D}-\mathrm{H} \cdots \mathrm{A} /{ }^{\circ}$ \\
\hline N7-H7A...O5 & 0.86 & 1.95 & $2.790(3)$ & 163.9 \\
\hline N7-H7B...N4 & 0.86 & 2.45 & 2.993(3) & 121.8 \\
\hline N7-H7B...O4 & 0.86 & 2.09 & $2.933(3)$ & 168.7 \\
\hline N8-H8A...N3 & 0.89 & 2.48 & $3.083(4)$ & 125.3 \\
\hline N8-H8B...N10 & 0.89 & 2.58 & $3.417(4)$ & 156.3 \\
\hline
\end{tabular}




\begin{tabular}{lllll}
\hline N9-H9...O4 & 0.86 & 2.15 & $2.954(3)$ & 155.5 \\
N10-H10...N2 & 0.86 & 2.34 & $3.005(3)$ & 134.8 \\
N11-H11A...N8 & 0.89 & 2.54 & $3.034(3)$ & 116.1 \\
N11-H11A... 3 & 0.89 & 2.02 & $2.845(3)$ & 153.6 \\
N11-H11B...O3 & 0.89 & 1.97 & $2.860(3)$ & 173.9 \\
N11-H11C...N1 & 0.89 & 2.08 & $2.971(3)$ & 175.5 \\
\hline
\end{tabular}

Table S16 Selected bond lengths $[\AA]$, bond angles $\left[{ }^{\circ}\right]$ and dihedral angles $\left[{ }^{\circ}\right]$ for compound $\mathbf{8}$

\begin{tabular}{|c|c|c|c|}
\hline C1-N7 & $1.3110(19)$ & $\mathrm{N} 1-\mathrm{C} 1-\mathrm{O} 1$ & $112.78(12)$ \\
\hline C1-N1 & $1.3149(19)$ & $\mathrm{N} 2-\mathrm{C} 2-\mathrm{N} 1$ & $116.16(13)$ \\
\hline $\mathrm{C} 1-\mathrm{O} 1$ & $1.3407(16)$ & $\mathrm{N} 2-\mathrm{C} 2-\mathrm{C} 3$ & $122.74(13)$ \\
\hline $\mathrm{C} 2-\mathrm{N} 2$ & $1.2963(18)$ & $\mathrm{N} 1-\mathrm{C} 2-\mathrm{C} 3$ & $121.09(12)$ \\
\hline $\mathrm{C} 2-\mathrm{N} 1$ & $1.3711(18)$ & $\mathrm{N} 3-\mathrm{C} 3-\mathrm{C} 4$ & $109.61(13)$ \\
\hline $\mathrm{C} 2-\mathrm{C} 3$ & $1.460(2)$ & $\mathrm{N} 3-\mathrm{C} 3-\mathrm{C} 2$ & $119.65(13)$ \\
\hline $\mathrm{C} 3-\mathrm{N} 3$ & $1.2999(19)$ & $\mathrm{C} 4-\mathrm{C} 3-\mathrm{C} 2$ & $130.68(12)$ \\
\hline $\mathrm{C} 3-\mathrm{C} 4$ & $1.4375(19)$ & $\mathrm{N} 4-\mathrm{C} 4-\mathrm{N} 5$ & $131.32(13)$ \\
\hline $\mathrm{C} 4-\mathrm{N} 4$ & $1.3155(19)$ & $\mathrm{N} 4-\mathrm{C} 4-\mathrm{C} 3$ & $108.48(12)$ \\
\hline C4-N5 & $1.3757(19)$ & $\mathrm{N} 5-\mathrm{C} 4-\mathrm{C} 3$ & $120.19(12)$ \\
\hline $\mathrm{C} 5-\mathrm{N} 10$ & $1.298(2)$ & $\mathrm{N} 10-\mathrm{C} 5-\mathrm{N} 8$ & $110.90(15)$ \\
\hline $\mathrm{C} 5-\mathrm{N} 8$ & $1.340(2)$ & $\mathrm{N} 10-\mathrm{C} 5-\mathrm{H} 5$ & 124.6 \\
\hline C5-H5 & 0.9300 & N8-C5-H5 & 124.6 \\
\hline C6-N9 & $1.298(2)$ & N9-C6-N8 & $106.97(14)$ \\
\hline $\mathrm{C} 6-\mathrm{N} 8$ & $1.3220(18)$ & N9-C6-H6 & 126.5 \\
\hline C6-H6 & 0.9300 & N8-C6-H6 & 126.5 \\
\hline $\mathrm{N} 2-\mathrm{O} 1$ & $1.4325(16)$ & $\mathrm{C} 1-\mathrm{N} 1-\mathrm{C} 2$ & $102.21(11)$ \\
\hline $\mathrm{N} 3-\mathrm{O} 2$ & $1.3734(18)$ & $\mathrm{C} 2-\mathrm{N} 2-\mathrm{O} 1$ & $102.26(11)$ \\
\hline $\mathrm{N} 4-\mathrm{O} 2$ & $1.4011(16)$ & $\mathrm{C} 3-\mathrm{N} 3-\mathrm{O} 2$ & $105.71(12)$ \\
\hline N5-N6 & $1.3191(17)$ & $\mathrm{C} 4-\mathrm{N} 4-\mathrm{O} 2$ & $104.90(11)$ \\
\hline $\mathrm{N} 6-\mathrm{O} 4$ & $1.2441(16)$ & $\mathrm{N} 6-\mathrm{N} 5-\mathrm{C} 4$ & $116.27(12)$ \\
\hline $\mathrm{N} 6-\mathrm{O} 3$ & $1.2516(16)$ & O4-N6-O3 & $119.83(12)$ \\
\hline N7-H7A & 0.8600 & O4-N6-N5 & $124.24(12)$ \\
\hline N7-H7B & 0.8600 & O3-N6-N5 & $115.93(12)$ \\
\hline N8-N11 & $1.4067(16)$ & C1-N7-H7A & 120.0 \\
\hline N9-N10 & $1.352(2)$ & C1-N7-H7B & 120.0 \\
\hline N9-H9 & 0.9212 & H7A-N7-H7B & 120.0 \\
\hline N11-H11A & 0.8703 & C6-N8-C5 & $106.86(13)$ \\
\hline N11-H11B & 0.8651 & C6-N8-N11 & $124.14(13)$ \\
\hline O5-H5A & 0.7917 & C5-N8-N11 & $129.00(13)$ \\
\hline O5-H5B & 0.8113 & C6-N9-N10 & $111.20(13)$ \\
\hline N8-N11-H11A & 107.4 & C6-N9-H9 & 128.2 \\
\hline N8-N11-H11B & 105.9 & N10-N9-H9 & 119.5 \\
\hline H11A-N11-H11B & 105.6 & C5-N10-N9 & $104.06(14)$ \\
\hline $\mathrm{C} 1-\mathrm{O} 1-\mathrm{N} 2$ & $106.59(10)$ & O3-N6-N5-C4 & $-179.980(124)$ \\
\hline N3-O2-N4 & $111.30(11)$ & $\mathrm{N} 4-\mathrm{C} 4-\mathrm{C} 3-\mathrm{C} 2$ & $177.237(148)$ \\
\hline H5A-O5-H5B & 118.5 & $\mathrm{O} 2-\mathrm{N} 3-\mathrm{C} 3-\mathrm{C} 2$ & $-177.941(127)$ \\
\hline N7-C1-N1 & $129.20(14)$ & $\mathrm{N} 2-\mathrm{C} 2-\mathrm{N} 1-\mathrm{C} 1$ & $-0.27(17)$ \\
\hline N7-C1-O1 & $118.02(13)$ & & \\
\hline
\end{tabular}

Table S17 Hydrogen bonds present in compound 8

\begin{tabular}{|c|c|c|c|c|}
\hline D-H $\cdots A$ & D-H/Å & $\mathrm{H} \cdots \mathrm{A} / \AA$ & $\mathrm{D} \cdots \mathrm{A} / \AA$ & $\mathrm{D}-\mathrm{H}^{\cdots} \mathrm{A} /{ }^{\circ}$ \\
\hline N7-H7A...O5 & 0.86 & 2.05 & $2.887(2)$ & 164.7 \\
\hline N7-H7B...N6 & 0.86 & 2.67 & $3.5037(18)$ & 163.3 \\
\hline N7-H7B...O3 & 0.86 & 2.55 & $3.2476(18)$ & 139.3 \\
\hline N7-H7B...O4 & 0.86 & 2.09 & $2.9182(18)$ & 162.0 \\
\hline N9-H9...N1 & 0.92 & 1.86 & $2.7755(17)$ & 175.9 \\
\hline N11-H11A...N4 & 0.87 & 2.39 & $3.0686(19)$ & 135.0 \\
\hline N11-H11A...O4 & 0.87 & 2.19 & $3.0116(18)$ & 156.7 \\
\hline N11-H11B...N5 & 0.87 & 2.43 & $3.2671(19)$ & 162.4 \\
\hline N11-H11B...O1 & 0.87 & 2.52 & $2.9867(16)$ & 114.3 \\
\hline O5-H5A...O3 & 0.79 & 2.20 & $2.9088(19)$ & 149.9 \\
\hline O5-H5B...O5 & 0.81 & 2.37 & $2.849(3)$ & 118.9 \\
\hline C5-H5 ...N2 & 0.93 & 2.59 & $3.389(2)$ & 145.0 \\
\hline $\mathrm{C} 5-\mathrm{H} 5 \ldots \mathrm{O} 3$ & 0.93 & 2.53 & $3.109(2)$ & 120.6 \\
\hline
\end{tabular}

\section{IR and NMR spectra}




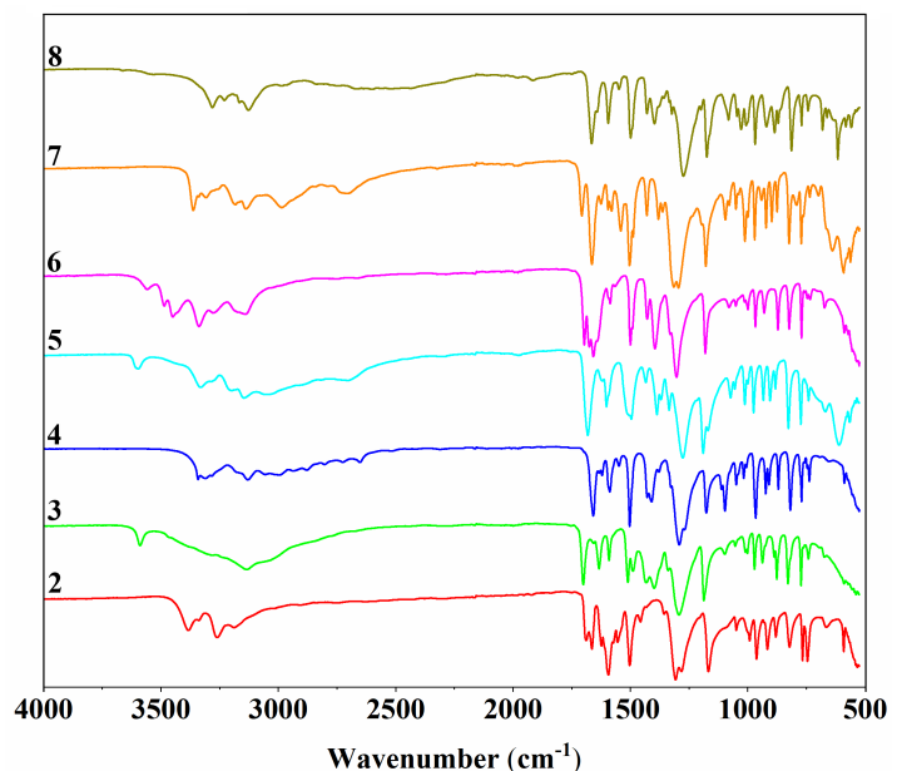

Figure S4 IR spectra for 2-8.

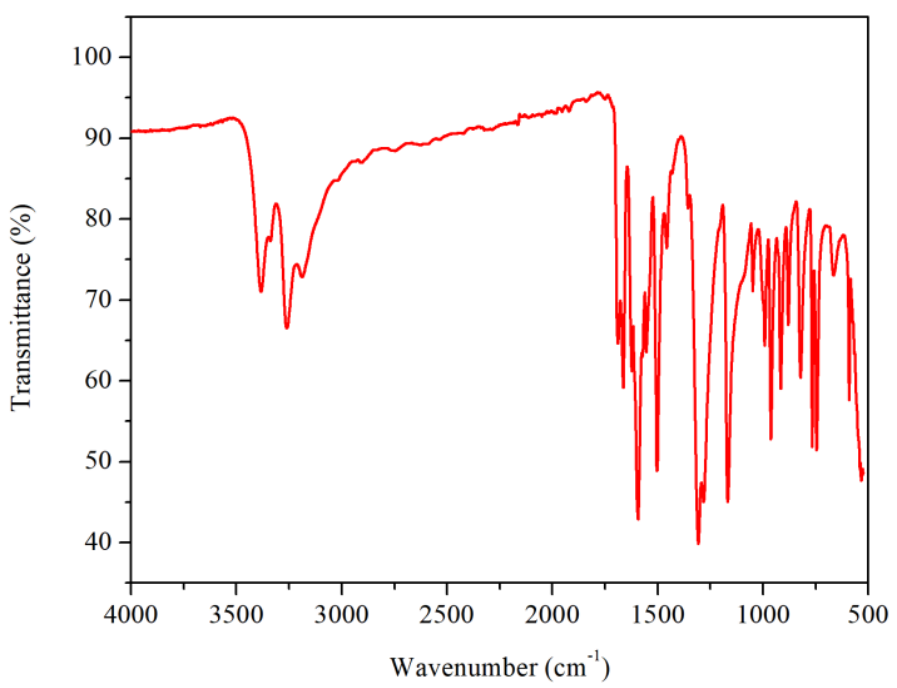

Figure S5 IR spectra for 2.
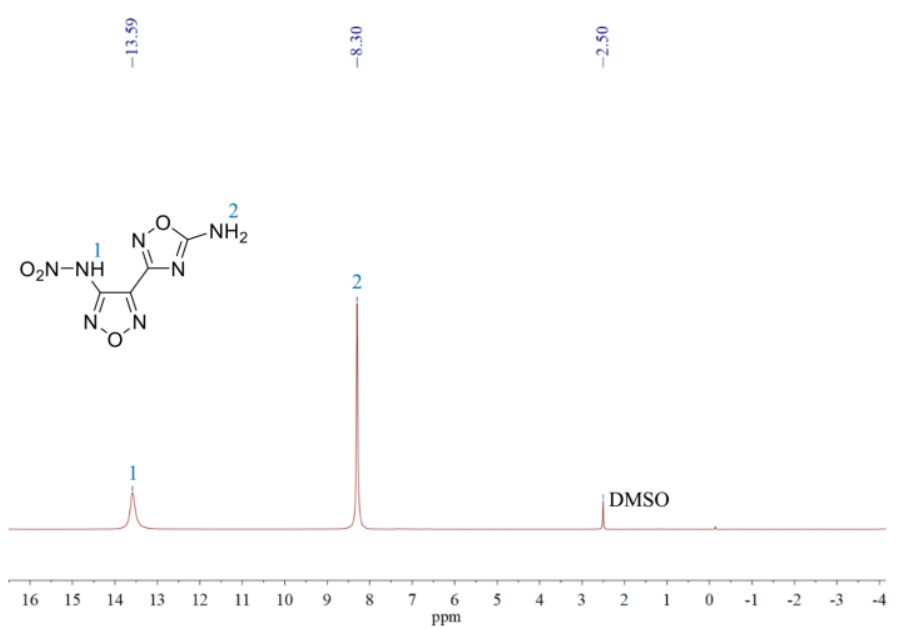

Figure S6 ${ }^{1} \mathrm{H}$ NMR spectra in DMSO- $d_{6}$ for $\mathbf{2}$ 


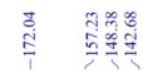

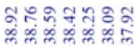
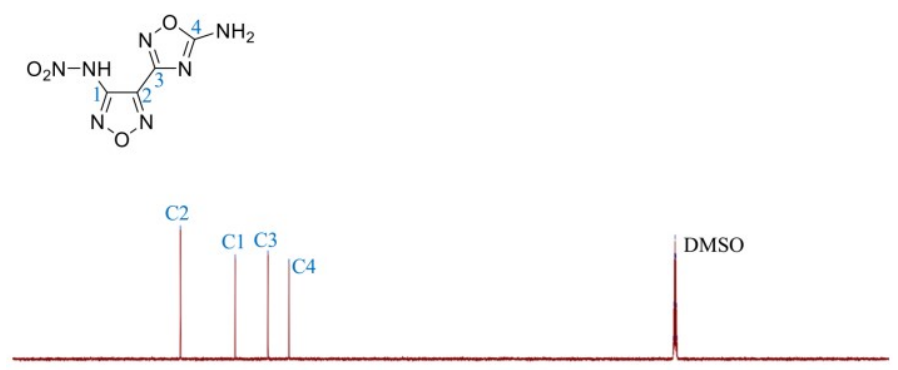

\begin{tabular}{llllllllllllllllllllllll}
\hline 210 & 200 & 190 & 180 & 170 & 160 & 150 & 140 & 130 & 120 & 110 & $\begin{array}{l}100 \\
\mathrm{ppm}\end{array}$ & 90 & 80 & 70 & 60 & 50 & 40 & 30 & 20 & 10 & 0 & -10
\end{tabular}

Figure $\mathbf{S} 7{ }^{13} \mathrm{C}$ NMR spectra in DMSO- $d_{6}$ for $\mathbf{2}$

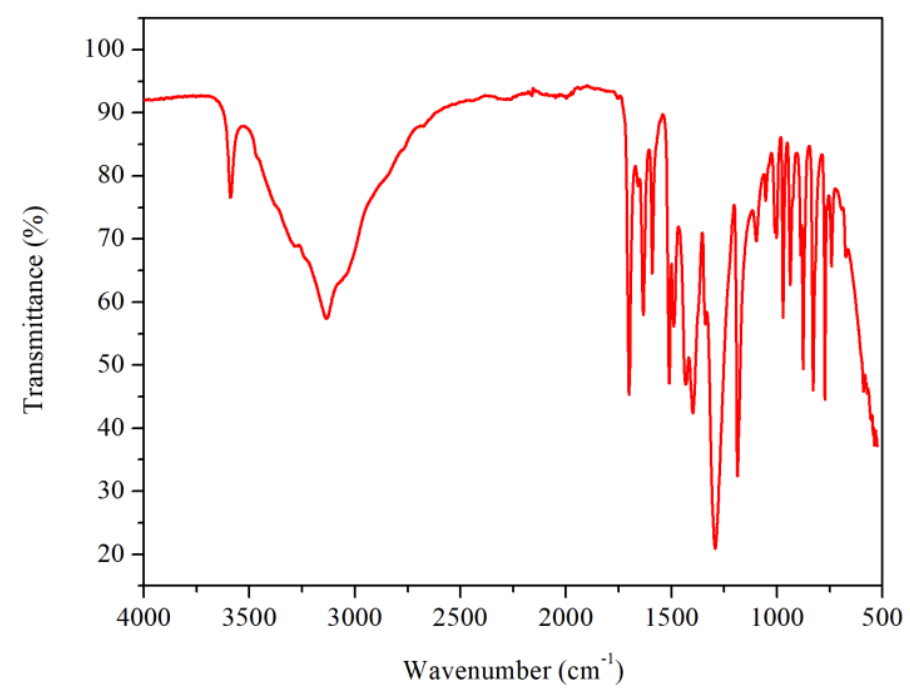

Figure S8 IR spectra for 3.
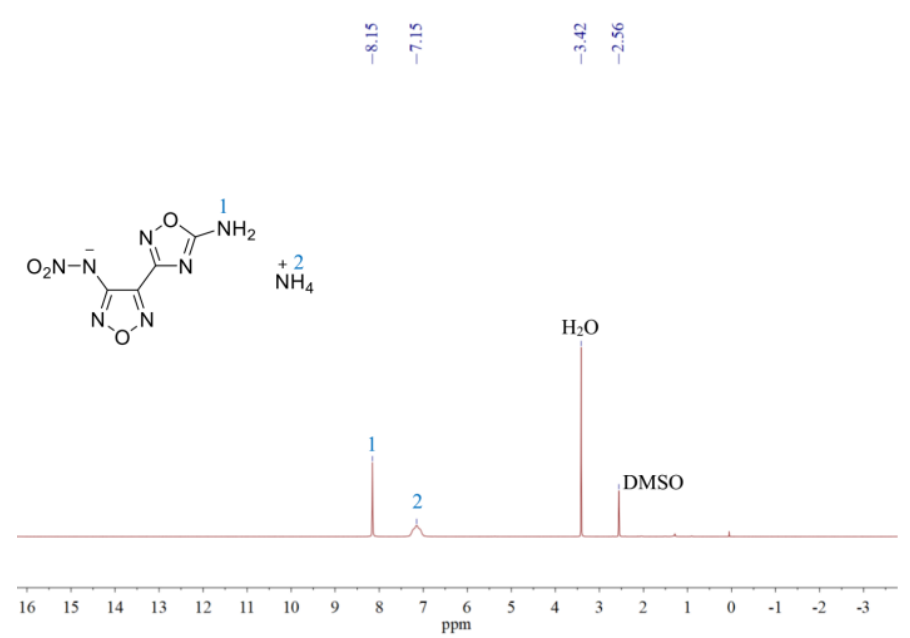

Figure S9 ${ }^{1} \mathrm{H}$ NMR spectra in DMSO- $d_{6}$ for 3 


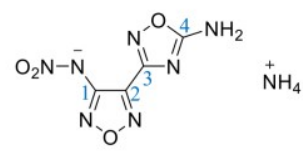

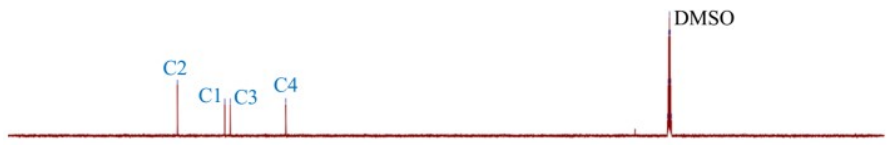

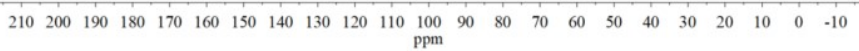

Figure S10 ${ }^{13} \mathrm{C}$ NMR spectra in DMSO $-d_{6}$ for 3

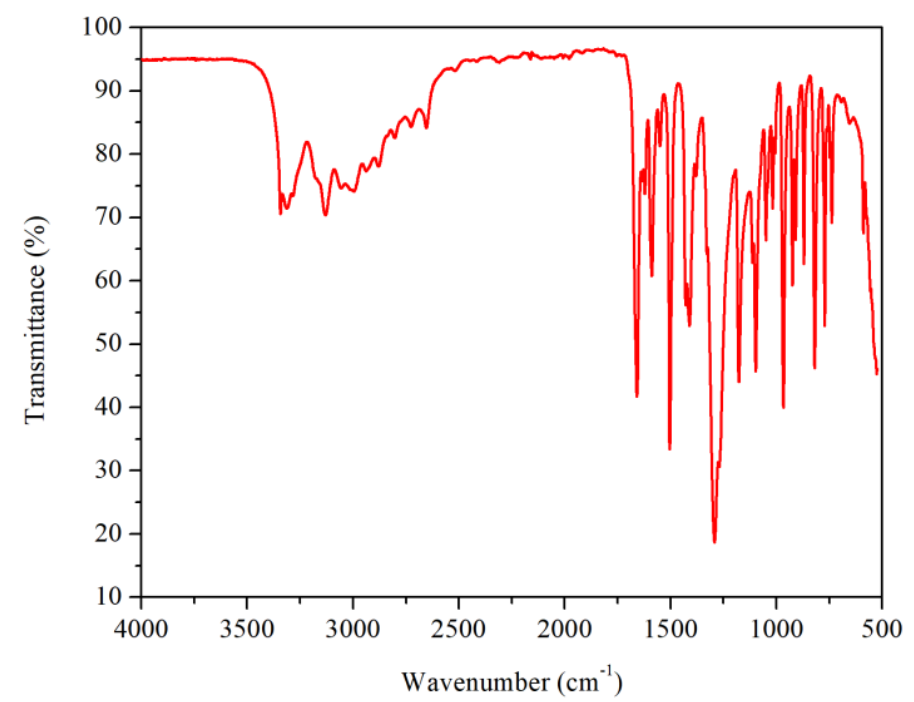

Figure S11 IR spectra for 4
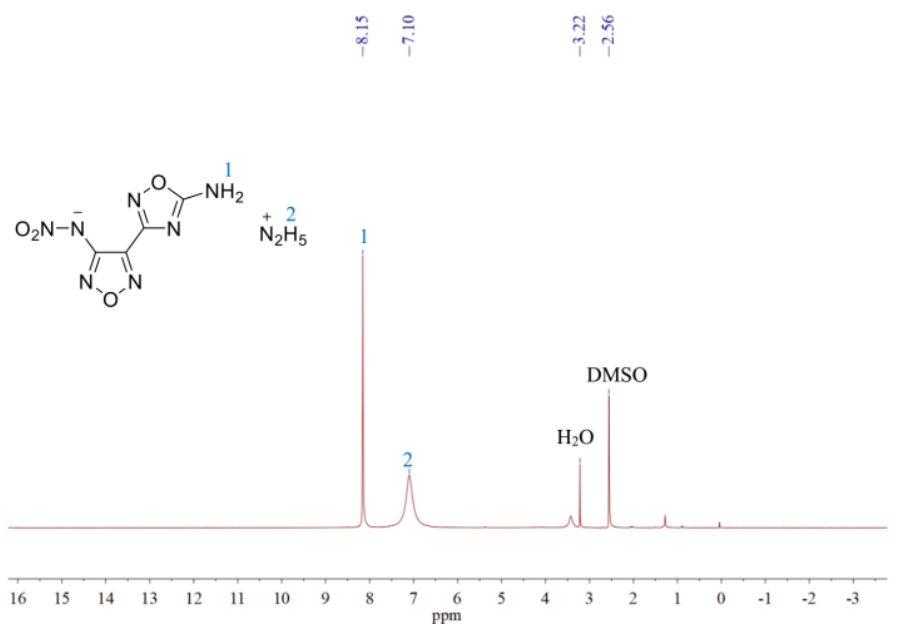

Figure S12 ${ }^{1} \mathrm{H}$ NMR spectra in DMSO- $d_{6}$ for 4 . 


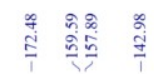

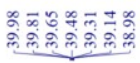

Ond

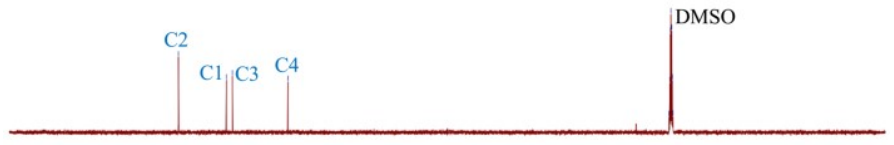

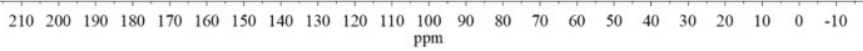

Figure $\mathrm{S} 13{ }^{13} \mathrm{C}$ NMR spectra in DMSO- $d_{6}$ for 4

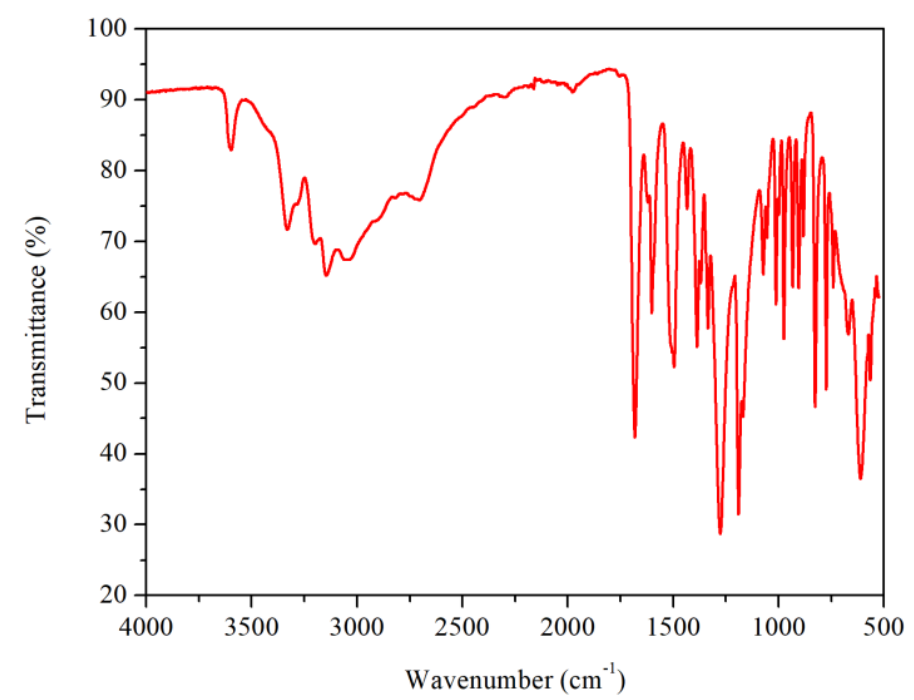

Figure S14 IR spectra for 5
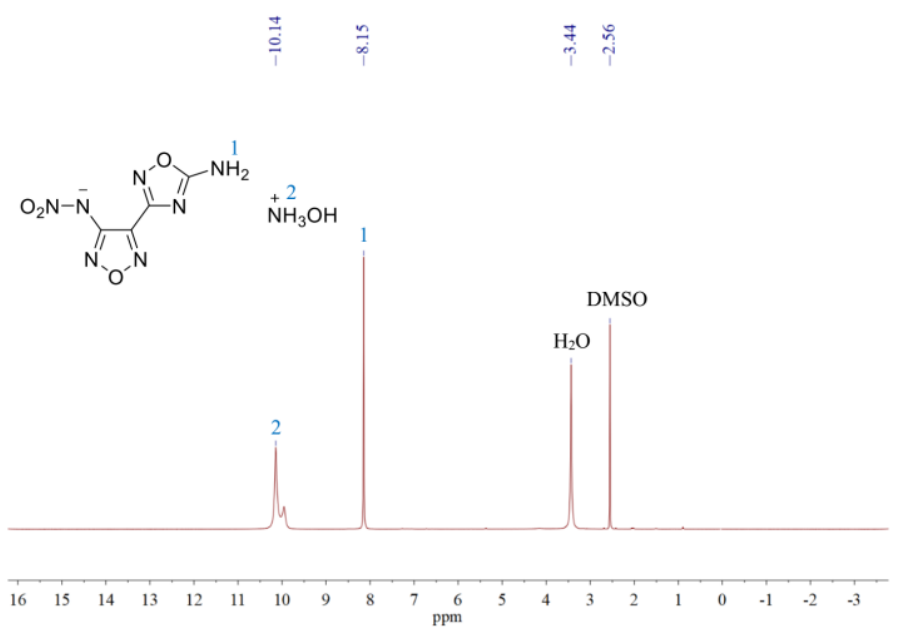

Figure S15 ${ }^{1} \mathrm{H}$ NMR spectra in DMSO- $d_{6}$ for 5 . 


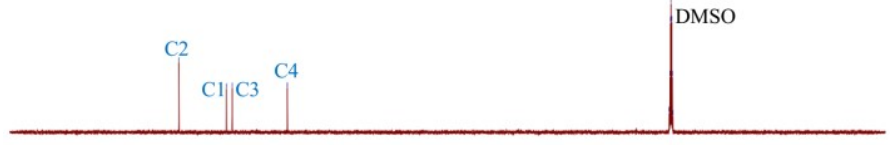

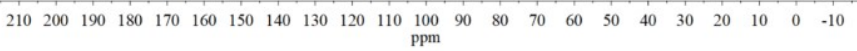

Figure S16 ${ }^{13} \mathrm{C}$ NMR spectra in DMSO $-d_{6}$ for 5 .

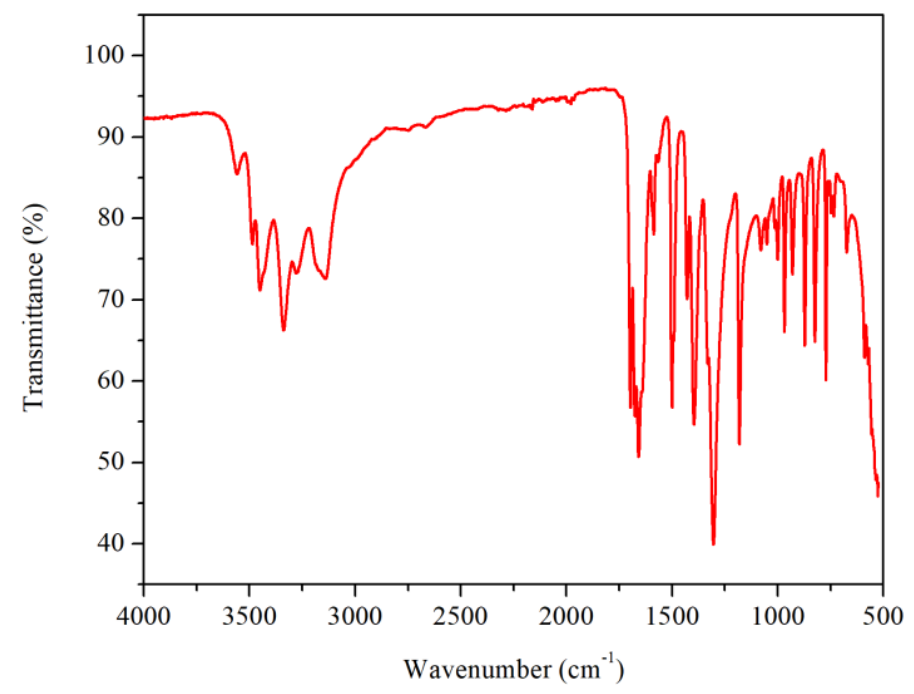

Figure S17 IR spectra for 6

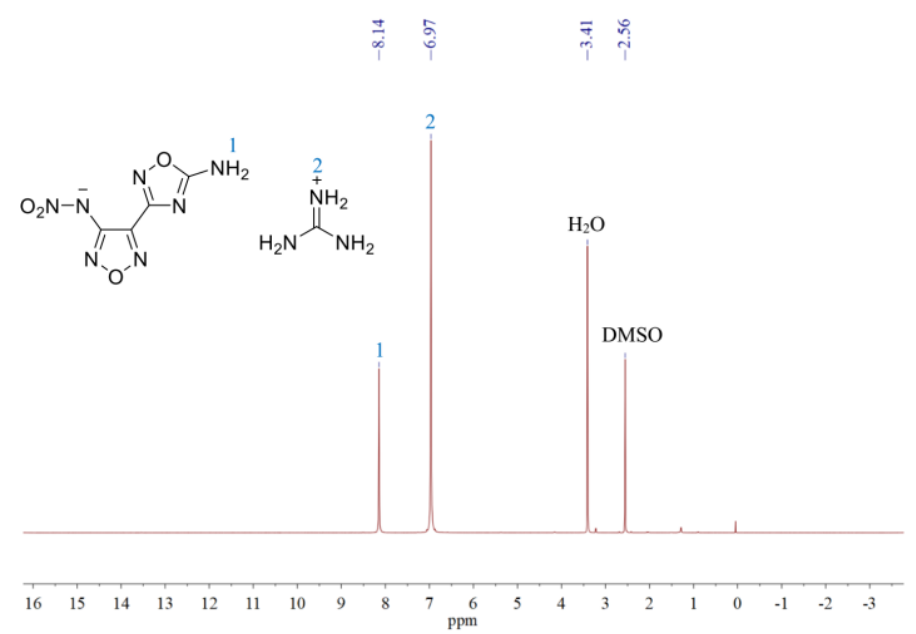

Figure S18 ${ }^{1} \mathrm{H}$ NMR spectra in DMSO- $d_{6}$ for 6 . 


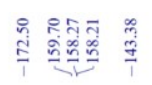

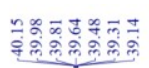

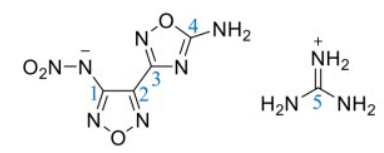

DMSO

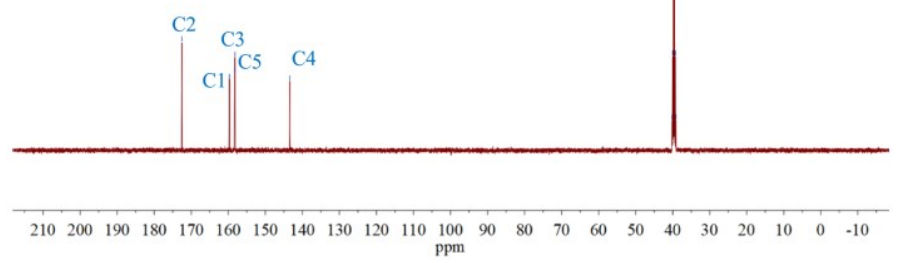

Figure S19 ${ }^{13} \mathrm{C}$ NMR spectra in DMSO- $d_{6}$ for 6 .

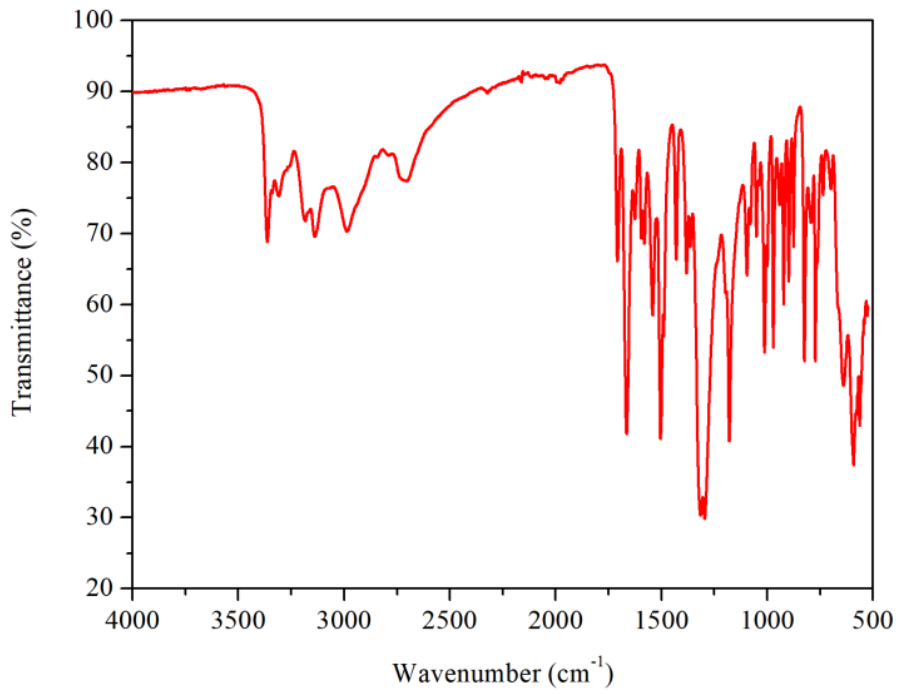

Figure S20 IR spectra for 7.

$\frac{n}{\infty}$

สุ
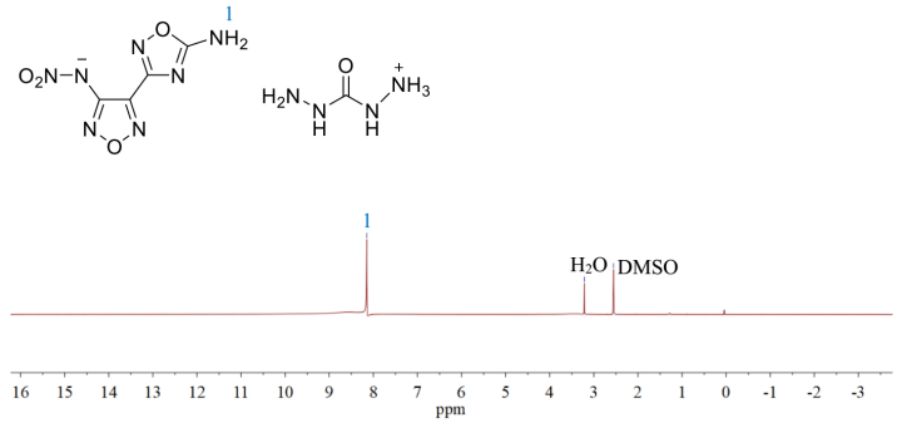

Figure S21 ${ }^{1} \mathrm{H}$ NMR spectra in DMSO- $d_{6}$ for 7 . 


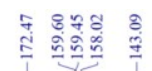
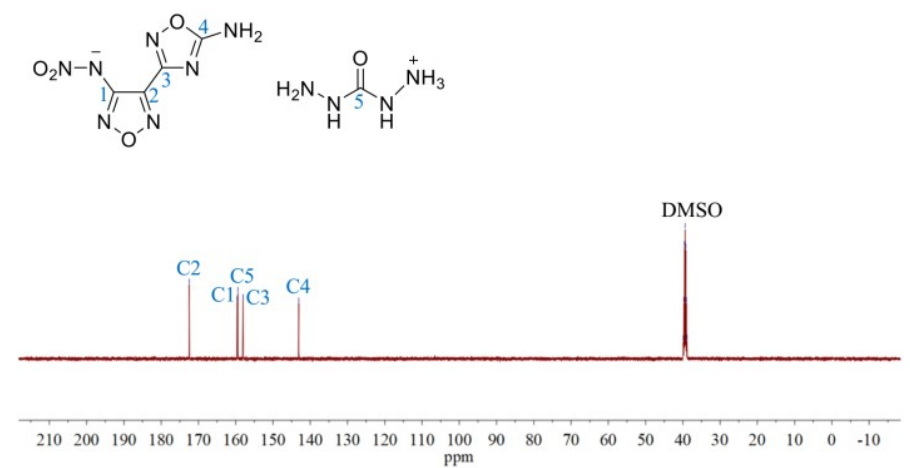

Figure S22 ${ }^{13} \mathrm{C}$ NMR spectra in DMSO- $d_{6}$ for 7.

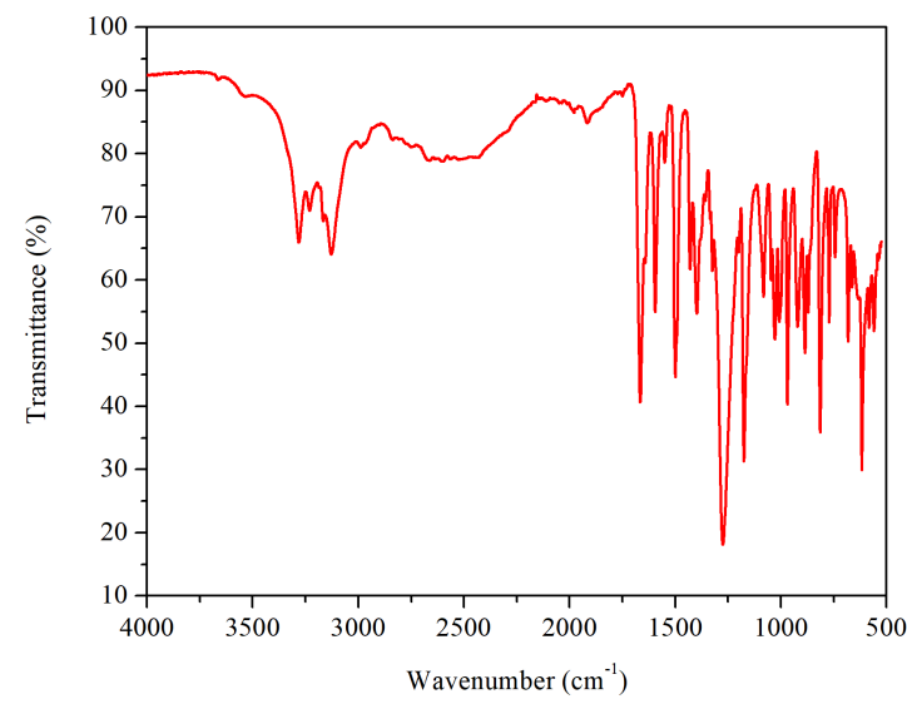

Figure S23 IR spectra for 8.

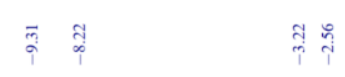

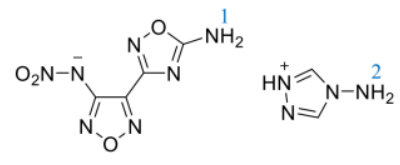

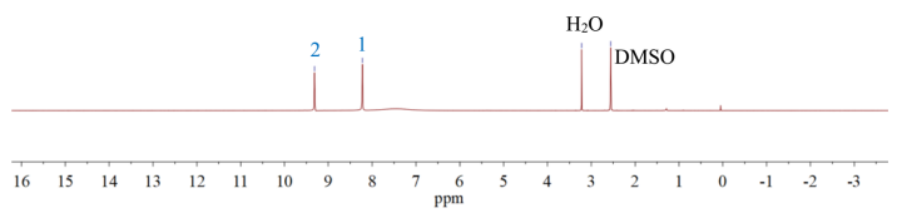

Figure S24 ${ }^{1} \mathrm{H}$ NMR spectra in DMSO- $d_{6}$ for $\mathbf{8}$. 

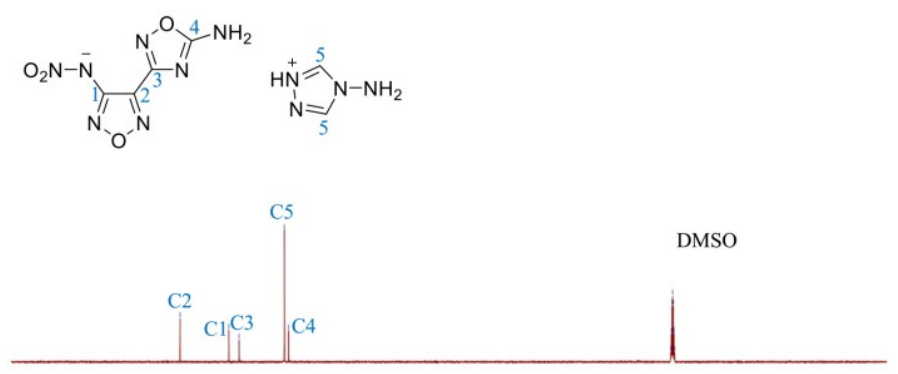

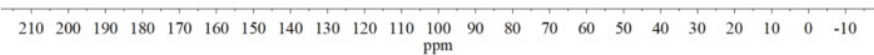

Figure $\mathbf{S 2 5}{ }^{13} \mathrm{C}$ NMR spectra in DMSO $-d_{6}$ for 8 .

\section{DSC and TG curves}

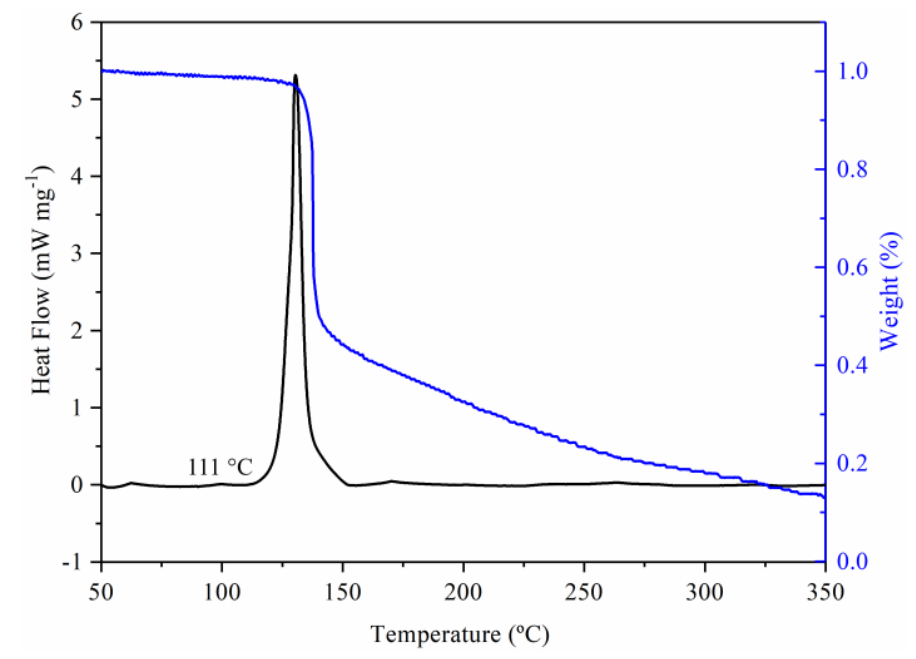

Figure S26 TG and DSC curves of 2 measured at a heating rate of $5{ }^{\circ} \mathrm{C} \mathrm{min}^{-1}$ (exo up).

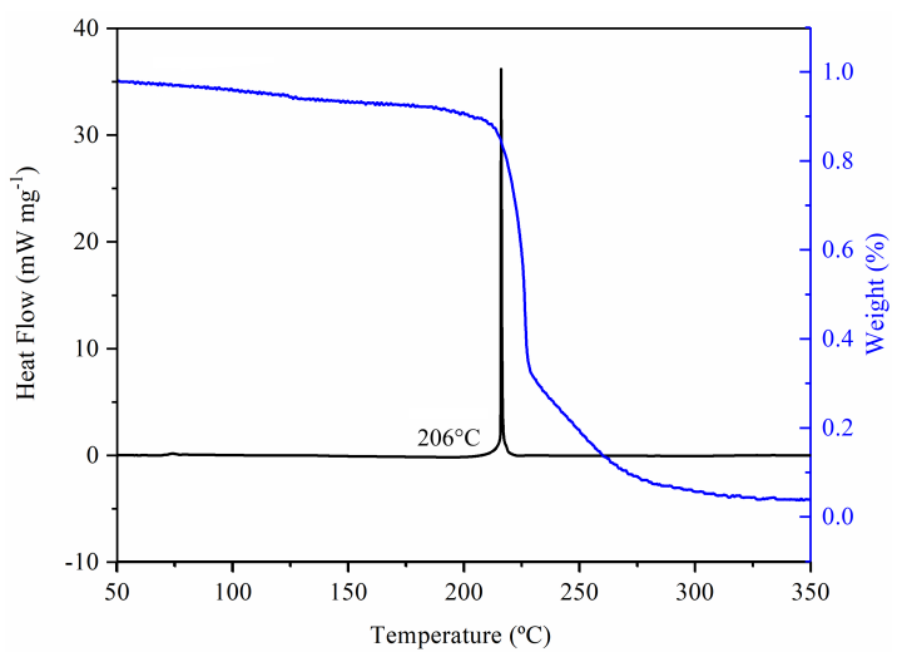

Figure S27 TG and DSC curves of 3 measured at a heating rate of $5^{\circ} \mathrm{C} \mathrm{min}^{-1}$ (exo up). 


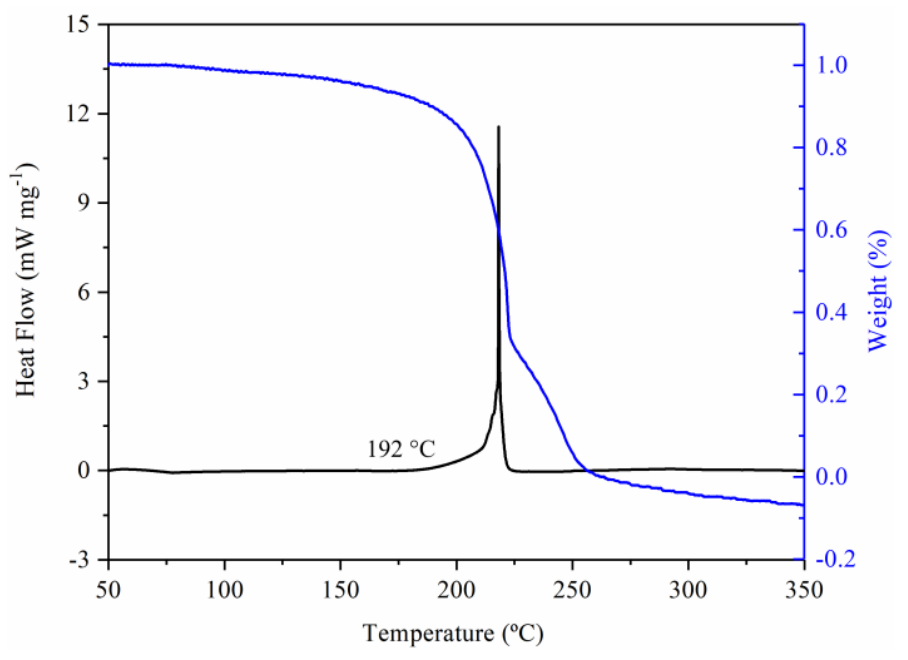

Figure S28 TG and DSC curves of 4 measured at a heating rate of $5{ }^{\circ} \mathrm{C} \min ^{-1}$ (exo up).

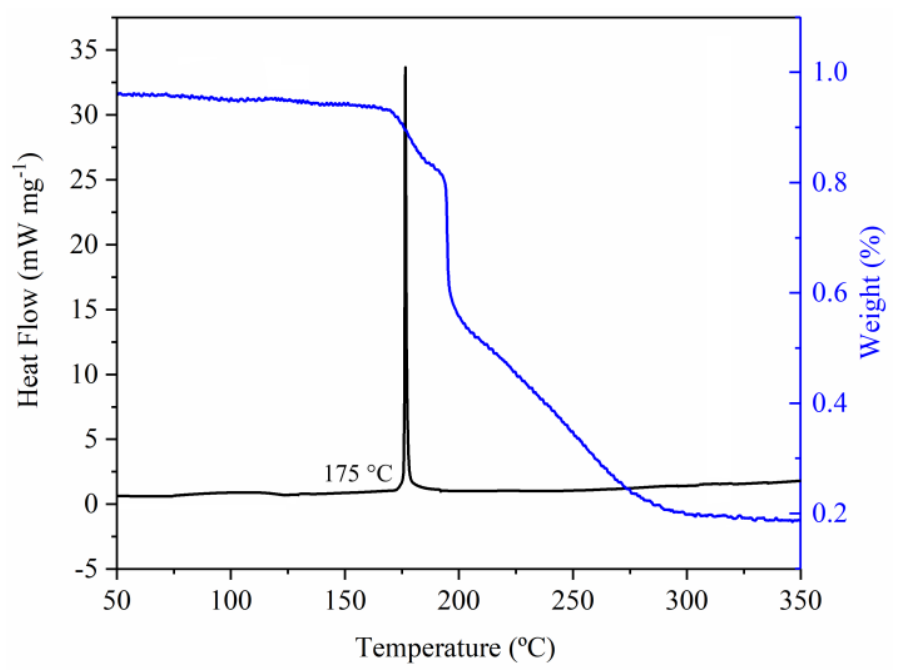

Figure S29 TG and DSC curves of 5 measured at a heating rate of $5{ }^{\circ} \mathrm{C} \min ^{-1}$ (exo up).

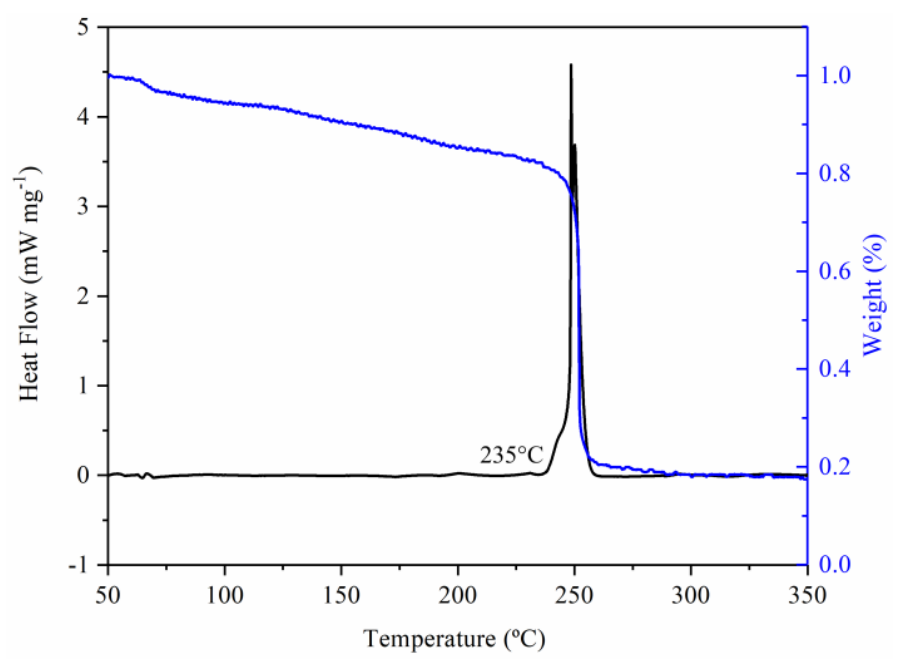

Figure S30 TG and DSC curves of 6 measured at a heating rate of $5{ }^{\circ} \mathrm{C} \min ^{-1}$ (exo up). 


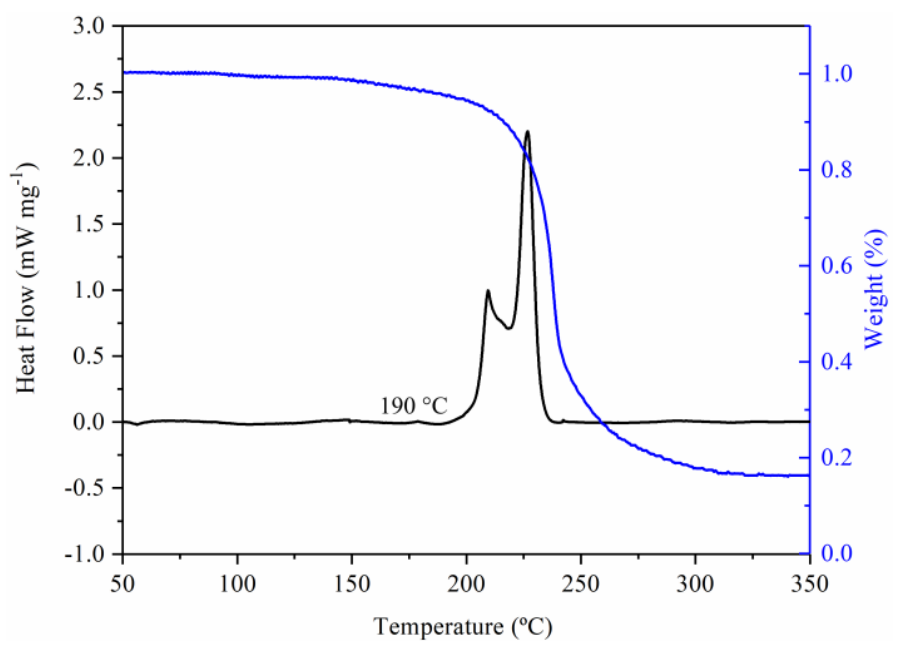

Figure S31 TG and DSC curves of 7 measured at a heating rate of $5^{\circ} \mathrm{C} \min ^{-1}$ (exo up).

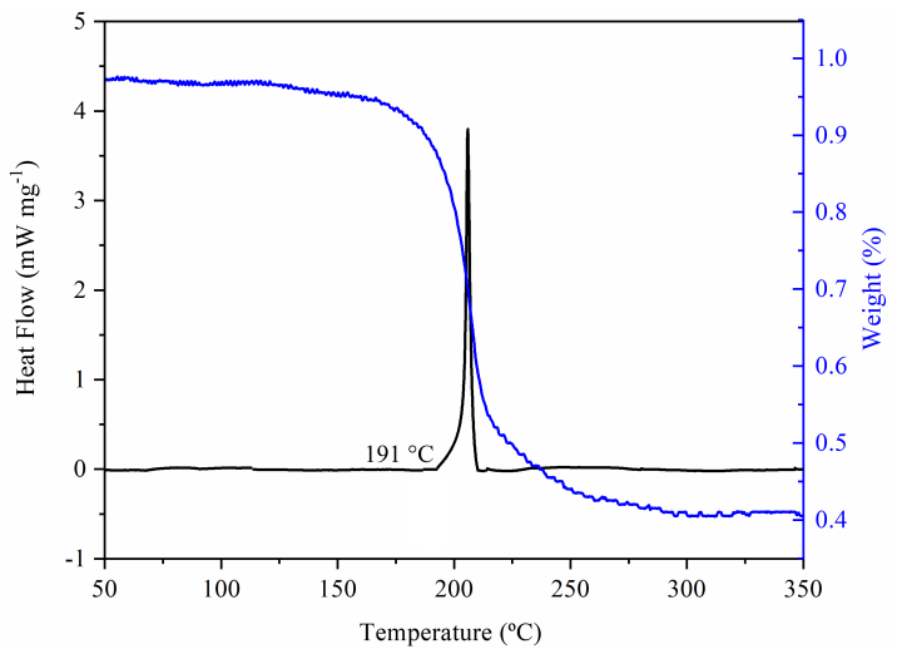

Figure S32 TG and DSC curves of 8 measured at a heating rate of $5^{\circ} \mathrm{C} \mathrm{min}^{-1}$ (exo up).

\section{XRD spectra}

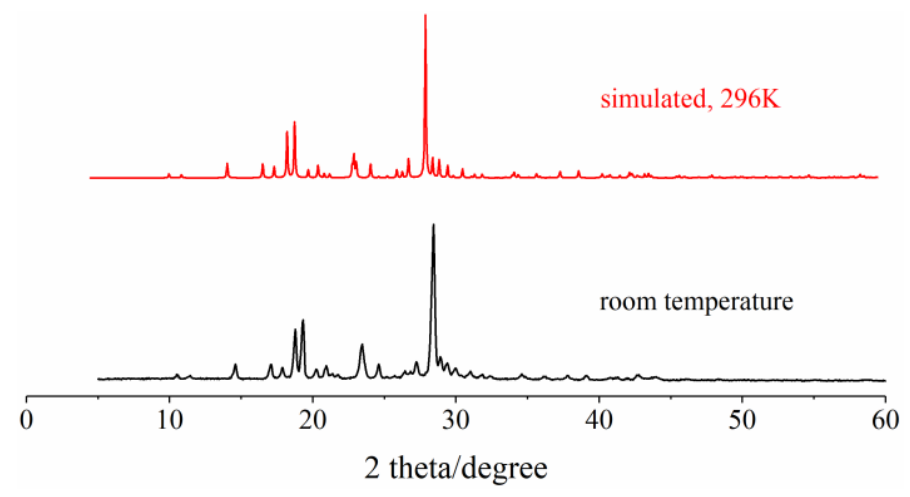

Figure S33 Powder X-ray diffraction pattern of $\mathbf{2}$ (black) and the calculated pattern (red) from the single crystal structure of 2. 


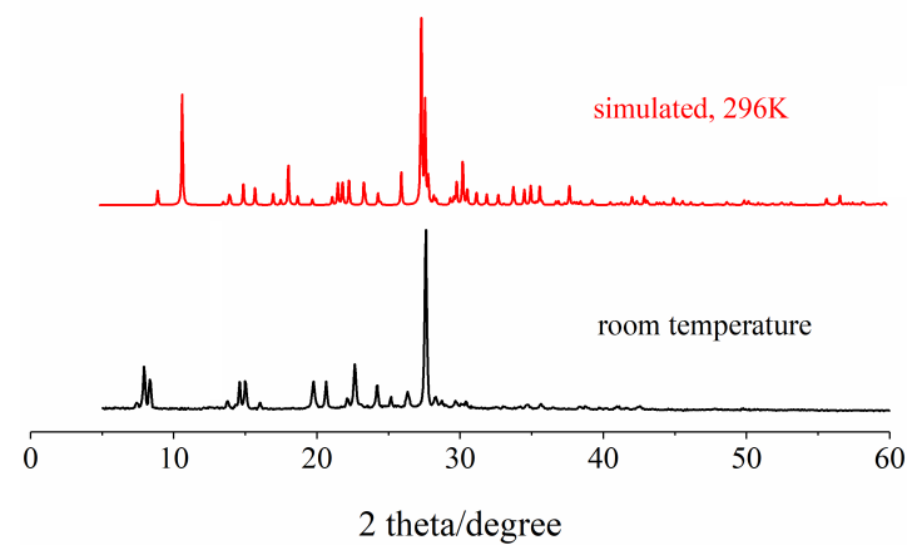

Figure S34 Powder X-ray diffraction pattern of 3 (black) and the calculated pattern (red) from the single crystal structure of 3.

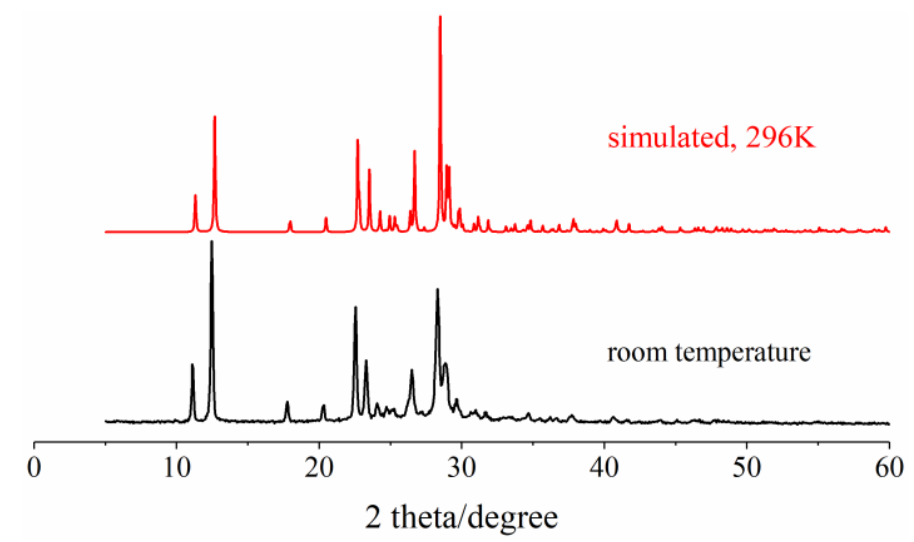

Figure S35 Powder X-ray diffraction pattern of 4 (black) and the calculated pattern (red) from the single crystal structure of 4.

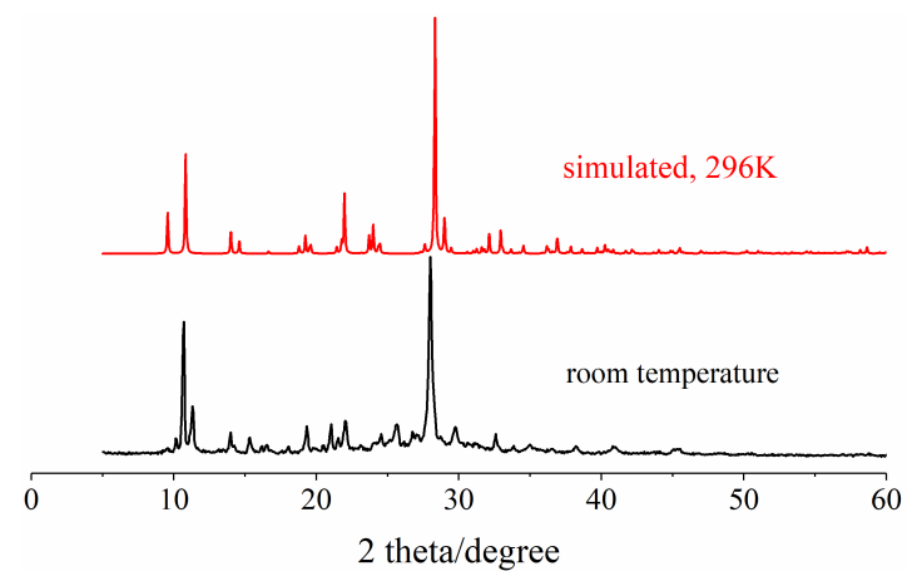

Figure S36 Powder X-ray diffraction pattern of $\mathbf{5}$ (black) and the calculated pattern (red) from the single crystal structure of $\mathbf{5}$. 


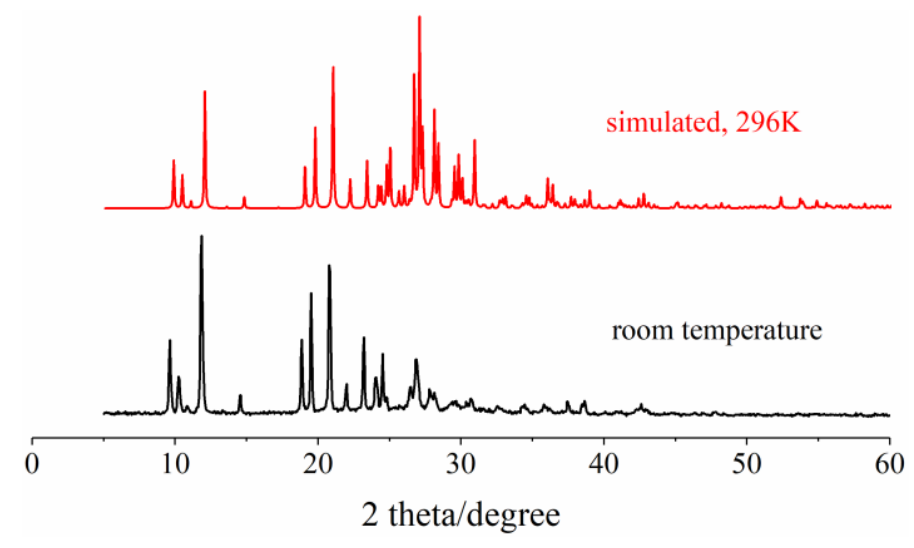

Figure S37 Powder X-ray diffraction pattern of 6 (black) and the calculated pattern (red) from the single crystal structure of 6.

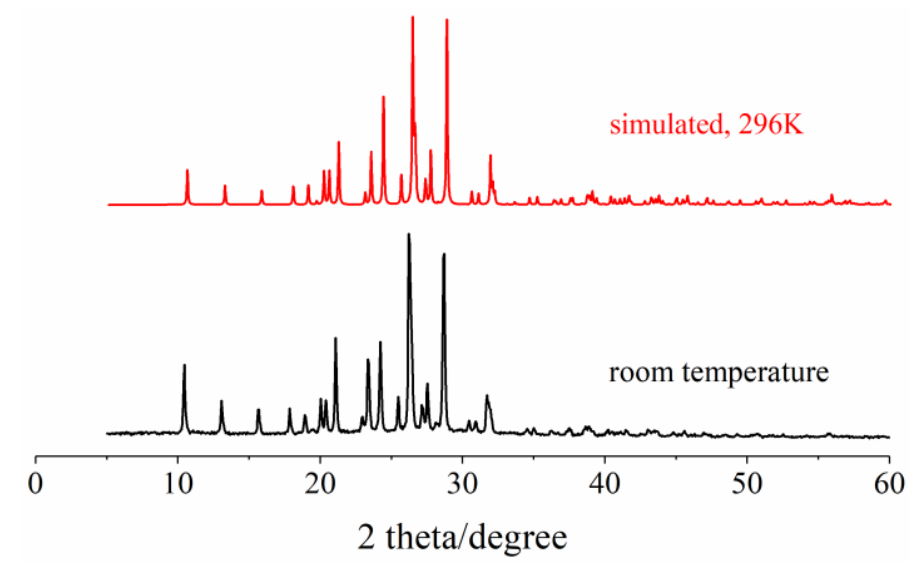

Figure S38 Powder X-ray diffraction pattern of 7 (black) and the calculated pattern (red) from the single crystal structure of 7.

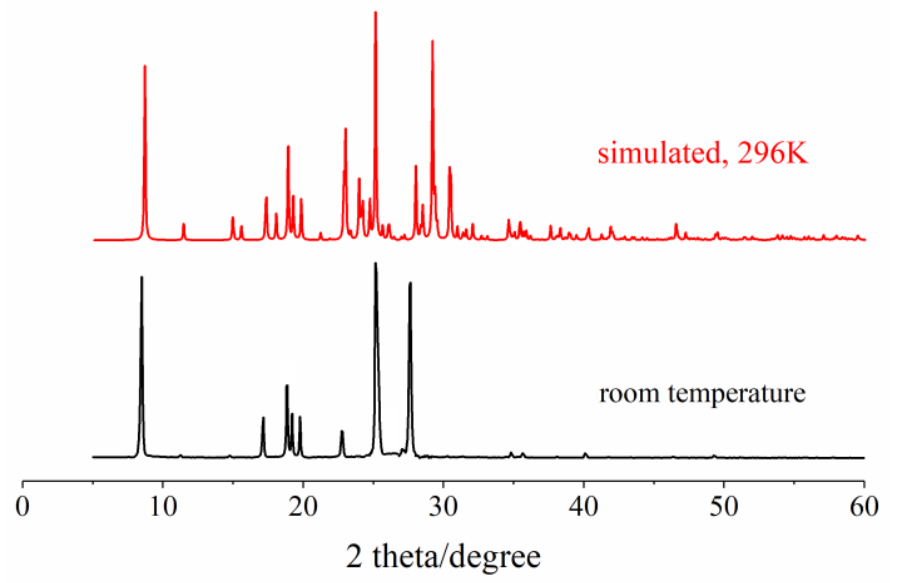

Figure S39 Powder X-ray diffraction pattern of $\mathbf{8}$ (black) and the calculated pattern (red) from the single crystal structure of $\mathbf{8}$.

\section{References}

1 M. J. Frisch, G. W. Trucks, H. B. Schlegel, G. E. Scuseria, M. A. Robb, J. R. Cheeseman, V. G.Zakrzewski, J. A. Montgomery, R. E. Stratmann, J. C. Burant, S. Dapprich, J. M. Millam, A. D.Daniels, K. N. Kudin, M. C. Strain, O. Farkas, J. Tomasi, V. Barone, M. Cossi, R. Cammi, B.Mennucci, C. Pomelli, C. Adamo, S. Clifford, J. Ochterski, G. A. Petersson, P. Y. Ayala, Q. Cui, K. Morokuma, D. K. Malick, A. D. Rabuck, K. Raghavachari, J. B. Foresman, J. Cioslowski, J. V. Ortiz, A. G. Baboul, B. B. Stefanov, G. Liu, A. Liashenko, P. Piskorz, I. Komaromi, R. Gomperts, R. L. Martin, D. J. Fox, T. Keith, M. A. Laham, C. Y. Peng, A. Nanayakkara, C. Gonzalez, M. Challacombe, P. M. W. Gill, B. Johnson, W. Chen, M. W. Wong, J. L. Andres, C. Gonzalez, M. Head-Gordon, E. S. Replogle and J. A. Pople, Gaussian 09, revision A. 01; Gaussian, Inc.: Wallingford, CT, 2009.

2 (a) A. D. Becke, J. Chem. Phys., 1993, 98, 5648-5652; (b) P. J. Stephens, F. J. Devlin, C. F. Chabalowski, M. J. Frisch, J. Phys. Chem., 1994, 98, $11623-11627$.

3 P. C. Hariharan and J. A. Pople, Theor. Chim. Acta., 1973, 28, 213-222.

4 J. W. Ochterski, G. A. Petersson, J. A. Montgomery, J. Chem. Phys., 1996, 104, 2598-2619. 
5 H. D. B. Jenkins, D. Tudela, L. Glasser, Inorg. Chem., 2002, 41, 2364-2367

6 (a) F. Trouton and Philos. Mag. 1884, 18, 54-57; (b) M. S. Westwell, M. S. Searle, D. J. Wales and D. H. Williams, J. Am. Chem. Soc., 1995, 117, 5013-5015. 